\title{
Von Mithradates von Kios bis Mithradates V. Euergetes. Kritische Bemerkungen zu Duane Rollers „Empire of the Black Sea“**
}

Altay Coşkun

\author{
Dem Andenken \\ meines Freundes \\ Alexandru Avram \\ († 4. August 2021) \\ in dankbarer Erinnerung \\ gewidmet
}

\section{Abstract}

With Empire of the Black Sea: the Rise and Fall of the Mithridatic World (2020), Duane Roller has published the first English monograph meant to cover the Pontic Kingdom of the Mithradatid dynasty. Although he falls short of presenting an up-to-date bibliography, the book is likely to become an influential reference work. The present chapter aims at closing several of the bibliographical gaps, by surveying recent (and forthcoming) scholarship especially on the kingdom's history prior to Mithradates VI Eupator. Topics include the Achaemenid ancestry of Mithradates of Kios, the flight of Mithradates I Ktistes to Kimiata, the role of the Galatians in the rise of the kingdom, the historicity of Mithradates III, the wars and diplomacy of Pharnakes I, the putative sibling marriage of Mithradates IV, chronological aspects of the rule of Mithradates V, and the continuity of Mithradatid foreign policies.

\section{Einführung}

Duane Roller ist einer der führenden Vertreter der antiken Geographie, der - vor allem seit seiner Emeritierung als Professor of Classics an der Ohio State University (2007) - mit geradezu schwindelerregender Schnelligkeit Bücher auf den Markt bringt. Seine Forschungen $\mathrm{zu}$ den Geographen Eratosthenes und Strabon werden durch sein Interesse an der Politik und den kulturellen Leistungen der Herrscherhäuser aus dem Hellenismus und der frühen Kaiserzeit organisch ergänzt. Die intensive Beschäftigung mit dem Schwarzmeerraum und der mithradatidischen Dynastie wurden wohl durch seine Beschäftigungen mit Strabon angeregt, welcher in seiner Geographie Mithradates VI. Eupator keine geringere Aufmerksamkeit schenkt als Pompeius oder Augustus (allein Alexander der Große wird öfter angesprochen). Pontische Königinnen waren bereits in Rollers vorletztem Buch zu

\footnotetext{
* Entstanden ist diese Studie im Rahmen meines an der University of Waterloo angesiedelten Projekts Ethnic Identities and Diplomatic Affiliations along the Ancient Black Sea Littoral ( $7^{\text {th }}$ Century BC-7 ${ }^{\text {th }}$ Century CE), für dessen Förderung ich dem Social Sciences and Humanities Council of Canada meinen Dank aussprechen möchte.
} 
den Herrscherinnen der augusteischen Zeit (2018) von zentraler Bedeutung. ${ }^{1}$ Mit seinem jüngsten Buch Empire of the Black Sea: the Rise and Fall of the Mithridatic World (2020) hat Roller gewissermaßen ein ,Prequel' dieser pontischen Dynastiegeschichte vorgelegt.

Gleichbleibendes Kennzeichen von Rollers Büchern ist der elegante Brückenschlag zwischen Popular- und Fachwissenschaft. Er schreibt leicht zugänglich für ein breites internationales Publikum mit ganz unterschiedlichen Vorkenntnissen, weiß, ausgewählte Details geschickt in seine Darstellung einzuflechten, bleibt quellennah und liefert zudem einen nützlichen wissenschaftlichen Apparat. Zwar fallen in seinem jüngsten Buch die zwei Karten (xi) und der reduzierte Stammbaum (xiii) recht spärlich aus, aber dies wird durch den Stellen- (259-64) und Namenindex (265-77) wettgemacht.

Die Bibliographie ist international gehalten. In ihr überwiegt die englischsprachige Forschung, aber immerhin sind auch Beiträge in weiteren maßgeblichen westeuropäischen Sprachen vertreten; zudem wurden osteuropäische Kollegen, die mittlerweile auch auf Englisch publizieren, in kleiner Auswahl berücksichtigt. Trotzdem gibt es gravierende Lücken. Man vermisst gleich mehrere Standardwerke, welche Roller gewiss bekannt sind, aber aus nicht nachvollziehbaren Gründen außen vorbleiben. ${ }^{2}$ Bedauerlich ist ferner, dass sich der Autor mit der im letzten Vierteljahrhundert stark angewachsenen Forschungsliteratur kaum auseinandergesetzt hat. Selbst in der Bibliographie gelistete Titel wurden häufig nicht an den relevanten Stellen der Argumentation eingebunden.

Die Gelegenheit, einen Überblick über die sich rasant entwickelnde Forschungslandschaft zu bieten, wurde damit versäumt, und das Problembewusstsein verharrt wiederholt in engen Grenzen. Das wird vor allem an den zahlreichen Widersprüchen sichtbar, die dem Lektorat der Oxford University Press entgangen sind. Im Ganzen gewinnt man den Eindruck, dass das ursprüngliche Manuskript rund ein Vierteljahrhundert in der Schublade gelegen hatte, bevor es mit punktuellen Retouchierungen hastig in den Druck gegeben wurde. Angesichts der anhaltenden, ja wachsenden Faszination, die das Pontische Reich und allen voran Mithradates VI. Eupator auf die gegenwärtige Altertumskunde und auch darüber hinaus ausüben, ist dieses Vorgehen sehr bedauerlich.

Die Arbeit gliedert sich in zwei fast gleichgroße Teile. Mit den ersten Kapiteln dringt das Buch in eine tatsächliche Lücke vor. Es bietet erstmals eine zusammenhängende Geschichte der früheren Mithradatiden (9-93), die man in einer westeuropäischen Sprache bisher vergeblich gesucht hat. ${ }^{3}$ Der zweite Teil (97-212) behandelt Eupator. Zu Persönlichkeit, Herrschaft und Kriegen dieses Königs liegt zwar schon eine ansehnliche Literaturfülle vor, ${ }^{4}$ aber das bedeutet nicht, dass man nichts Neues mehr über ihn sagen könnte. Ganz im

\footnotetext{
${ }^{1}$ ROLLER 2010 (Eratosthenes); 2014 und 2018a (Strabon); 2006 und 2019 (weiteres zur historischen Geographie); 1998, 2003, 2004 (späthellenistische und frührömische Herrscher); 2018b (Königinnen der augusteischen Zeit, bes. 79-120 zu Dynamis und Pythodoris).

${ }^{2}$ Z.B. fehlen DE CALlataŸ 1999; ENGELS 1999; MÜLler 2010; BIFFI 2010; MuCCIOLI 2013.

${ }^{3}$ Vgl. die russische Monographie von SAPRYKIN 1996. Besser sieht die Situation für das Bosporanische Reich aus: GAJDUKEVIČ 1971; SAPRYKIN 2002; JAJLENKO 2010; KOZLOVSKAYA 2017; KUZNETSOV 2017; vgl. MiCHELS 2009, 87-103; ZAVOIKINA 2014.

${ }^{4}$ In Rollers Bibliographie fehlen z.B. GOUKOWSKY 2003; BURCU ERCIYAS 2006; HeINEN 2006A; MATYSZAK 2008; MAYOR 2010; BALLESTEROS PASTOR 2013 (obwohl zahlreiche seiner anderen Arbeiten bei Roller
} 
Gegenteil wächst das ihm oder seiner Herrschaftszeit gewidmete Schrifttum unaufhörlich an. ${ }^{5}$

Dagegen ist das Ende von Rollers Darstellung überraschend abrupt: Den Nachfolgern des Mithradates werden kaum zwei Seiten (212f.) eingeräumt, obwohl die Geschichte pontischer Reiche auf kleinasiatischem Boden noch länger als ein weiteres Jahrhundert andauerte, abgesehen davon, dass auch die Mithradatiden selbst vor allem unter Pharnakes II. sowie seinem Sohn Dareios (in Pontos) und seiner Tochter Dynamis (am Bosporos) weiterhin oder erneut wichtige Rollen spielten. Der Abbruch der Darstellung ist zwar im kurzen Vorwort angedeutet, in dem Eupator als ,last and most famous king“" (of Pontos) angesprochen wird (ix), aber die kurzen Herrschaftszeiten des Pharnakes II. und später des Dareios sowie der Aufstand seines Bruders Arsakes hätten sicher mehr Raum verdient. ${ }^{6}$ Zudem ergibt sich ein Widerspruch zu der von Roller geteilten (vor allem in Russland populären) Auffassung, dass mit Aspurgos, dem Nachfolger der Dynamis im Bosporanischen Reich, ein Sohn der Mithradates-Enkelin herrschte und er der Stammvater einer bis ins 4. Jh. n. Chr. reichenden Reihe von Königen sei. Träfe dies zu, dann hätte Rollers Buch ja weniger als die Hälfte der Mithradatidengeschichte behandelt. ${ }^{7}$

Allerdings ist diese Theorie weder mit den Quellen noch mit regulären biologischen Reproduktionsmustern vereinbar, wie in der rezenten Literatur immer wieder betont wird. Die Reihe der Mithradatiden endete also mit Dynamis ca. 8 n. Chr., ${ }^{8}$ trotz der anderslautenden Behauptung, die Tacitus dem Mithradates VIII. (38/39-ca. 45 n. Chr.) in den Mund legt: Dass dieser Sohn des Aspurgos und der Gepaipyris noch mit seiner achämenidischen Abstammung geprahlt habe, ist entweder eine Fiktion des Geschichtsschreibers, die Zeitgenossen angesichts seines pseudo-dynastischen Namens glaubhaft erschienen sein könnte,

aufgeführt sind); ferner z.B. Olshausen UND Biller 1984; MielCZAREK 1999; MacDonald 2005; BeKKER-NIELSEN 2006; MiCHELS 2009; MUCCIOLI 2013; ChANIOTIS 2018, 209-221. Der epigraphische Neufund zu Hypsikrateia ist erfasst (S. 193), aber zu ergänzen wären vor allem HEINEN 2008A; 2012; BALLESTEROS PASTOR 2011B; GABELKO 2013.

${ }^{5}$ Z.B. MAdSEn 2020; PAyen 2020, 224-394; Rendine 2020; Ballesteros Pastor 2020A; Braund, Peter

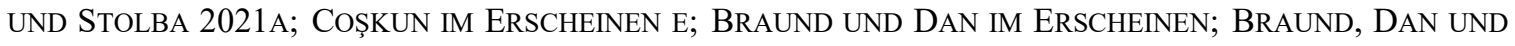
GEHRKE IM ERSCHEINEN.

${ }^{6}$ Pharnakes II.: [Caes.] BAlex. 34-78; App. Mithr. 120,590-595; Cass. Dio 42,45-47. Wenig ist über Dareios bekannt: App. BCiv. 5,75,319; vgl. Strab. 12,3,14. Noch schlechter ist es um dessen Bruder Arsakes bestellt: Strab. 12,3,38. Zusätzlich zu den von Roller gelisteten Arbeiten siehe zu Pharnakes II.: BALLESTEROS PASTOR 2008/18; 2017; 2021A; CoșKUN 2014B; 2016A; 2019B; 2019/20; ENGELS 2017, 60-65. Zu Dareios: PRIMO 2010, 162; SøRENSEN 2016, 121-126 (trotz fragwürdiger Prämissen zum Zustand von Pontos); COŞKUn 2021B, 250 und 253. Arsakes: Roller 2018A, 715f.; MADSEN 2020, 88. Roller 2020, 194 mit Anm. 10 streift auch Orsabaris, eine mögliche Tochter Eupators; weiteres hierzu unten in Anm. 30. Zu Dynamis siehe die nächsten drei Anm.

${ }^{7}$ ROLLER 2020, 213 und 215, aber unter Verzicht auf genealogische Details. Die angebliche Kontinuität setzt voraus, dass Dynamis entweder in vierter Ehe mit Aspurgos verheiratet (ROSTOVTZEFF 1919; MACDONALD 2005, 53f.) oder aber dessen Mutter war, wobei in letzterem Fall der inschriftlich als Asandrochos bezeugte Vater mit ihrem ersten Gemahl Asandros gleichgesetzt wird: so GAJDUKEVIČ 1971, 328-339; SAPRYKIN 2002, 97-99; 2005, 170f. sowie SAPRYKIN UND FEDOSEEV 2009; IVANTCHIK UND TOKHTAS'EV 2011, 165; ZAVOYKINA, NOVICHIKHIN UND KONSTANTINOV 2018, 683. Eine Variante erklärt ihren zweiten Gatten Scribonius zum Vater des Aspurgos: PARFENOV 1996.

${ }^{8}$ Siehe jetzt COŞKUN UND STERn 2021A, bes. 201-204. Vgl. HeINEN 2008A, 191-201; JAJLENKO 2010, $232-$ 249; AVRAM 2011; CoşKUN APR s.v. Dynamis [Addendum]. Zu Dynamis, Asandros und Scribonius ergänze - neben der in Anm. 3-7 genannten Literatur - BRAUND 2004; CoșKUN 2016A, 58-64 und 2019C. 
oder aber basiert auf einer sonst nicht überlieferten Tradition des iranischen Stammes des Aspurgos; seine Gattin wird jedenfalls gemeinhin als Thrakerin betrachtet und kommt für eine Abstammung von Mithradates kaum in Frage. ${ }^{9}$ Den Abschluss von Rollers Darstellung bilden knapp zwei Seiten zum Afterlife Eupators, auf denen einige Beispiele für seine Rezeption von der Antike bis in die Neuzeit, nicht aber in der Zeitgeschichte, geboten werden (215f.). ${ }^{10}$

Es ist davon auszugehen, dass vor allem Rollers Darstellung zu den frühen Mithradatiden häufig zur Hand genommen werden wird. Die hier vorgelegten bibliographischen Ergänzungen und weiterführenden kritischen Überlegungen sollen dazu dienen, Rollers pontische Dynastiegeschichte zu ergänzen, kritisch zu hinterfragen oder, wo nötig, auch zu korrigieren. Meine zahlreichen Bemerkungen fügen sich nun ihrerseits zu einer Art Abriss der frühen Familiengeschichte zusammen, ohne freilich mit einer Gesamtdarstellung rivalisieren oder in eine abschließende historische Würdigung münden zu wollen. Dennoch wird sich am Ende zumindest ein kleines Fazit ergeben, das die beachtliche Kontinuität dynastischer Politik bei den Mithradatiden herausstellen wird.

\section{Die achämenidische Abstammung der Mithradatiden}

Nach einer Einführung in den historischen Raum Pontos mitsamt ausgewählten Sehenswürdigkeiten und Legenden (9-24) wendet sich Roller recht ausführlich dem Dynastiegründer Mithradates I. Ktistes zu, der in Erwartung eines weniger spezialisierten Publikums „Mithradates the Founder“ genannt wird (25-39). Nur knapp zwei Seiten (25f.) werden der Frage nach dessen Ahnen aus der Blütezeit der Achämeniden gewidmet, die der Legende nach von Dareios I. oder einem anderen der sieben Verschwörer gegen den Usurpator des Jahres 522 v. Chr. abstammten. Roller kommt zu dem Urteil, dass jener dynastische Anspruch trotz bestehender Probleme plausibel sei. ${ }^{11}$ Dies mag zutreffen, doch hätte auch die detaillierte Quellenanalyse von Monica D’Agostini Berücksichtigung verdient, der zufolge die Mithradatiden zwar eine iranische Dynastenfamilie aus achämenidischer Zeit darstellten, aber deren Rückführung auf einen der Sieben von Hieronymos von Kardia erfunden wurde. Sicher entscheiden lässt sich die Frage nicht. ${ }^{12}$ Immerhin kann man feststellen, dass die genealogischen Ansprüche bis in späthellenistische Zeit weiter

\footnotetext{
${ }^{9} \mathrm{Zu}$ Mithradates VIII. siehe Tac. Ann. 12,18,2. Der Anspruch wird weitgehend anerkannt (z.B. GAJDUKEVIČ 1971, 340-343; ALIDOUST 2020, 327f.; BALLESTEROS PASTOR 2021, 186), trotz der zunehmenden Zweifel daran, dass Dynamis ein genealogisches Bindeglied sein kann (siehe vorangehende Anm.). Eine detailliertere Studie mit negativem Urteil wird derzeit von Germain Payen vorbereitet.

${ }^{10}$ Siehe jetzt JUNG-KAISER 2016.

${ }^{11}$ Siehe Polyb. 5,43,2; Diod. 19,40,2; auch Sall. Hist. F 2,73 M; Flor. 1,40,1; dazu RolLER 2020, 25f. (mit 221, Anm. 2-7) und 194 (mit 243, Anm. 12), mit Verweis auf LEROUGE-COHEN 2017; auch MCGING 1986B; Bosworth Und WheATley 1998; OlbryCht 2009; BALlesteros PASTOR 2012 und 2015, aber ohne Ausdifferenzierung der unterschiedlichen Positionen. Vgl. auch CANEPA 2020, 104.

12 D’Agostini 2016, bes. 92. PRIMO 2008 (vgl. 2009, 264) tritt besonders für den Informationsfluss über Hieronymos von Kardia ein; vgl. BosworTH Und WheATley 1998, 155; 163; D’AgOstini 2016, 87; 89; 91 ; COŞKUn In Begutachtung A. Kritisch äußern sich dagegen BALlesteros Pastor 2012, 367, Anm. 7 und OGDEN 2017, 92.
} 
gesteigert sowie die Abstammung einerseits von Kyros oder Dareios und andererseits von Alexander dem Großen und Seleukos I. Nikator postuliert wurden. ${ }^{13}$

Im Schlussteil greift Roller das Thema nochmals kurz auf und betont nun überzeugender, dass jedenfalls für Mithradates Eupator persische Ursprünge weniger wichtig gewesen seien als seine westliche Orientierung: „As the power of Rome increased, the Mithridatids became the champion of Greek ways against the invasive Romans, and when Mithridates VI took the surname Dionysos, who was an ancestor of Alexander the Great, he placed himself firmly in the Greek tradition." ${ }^{14}$ Aber weitere Überlegungen dazu, wann und warum ein achämenidischer Ursprung konstruiert oder propagiert worden sein könnte, unterbleiben. Hier hätte die jüngst intensiv geführte Diskussion über Persianism(us), also die weit verbreitete Erfindung oder zumindest Neukonstruktion achämenidischer Vorfahren und Traditionen in hellenistischer Zeit, eingehender ausgeführt werden können. Dabei hätte es sich angeboten, das Thema einerseits mit den Iranismus-Theorien vor allem der russischen, auf Michael Rostovtzeff basierenden Forschung und andererseits mit den zu Mithradates Eupator und Pharnakes II. geführten Debatten zu ihrem achämenidischen Erbe zu verbinden. Auch die Frage nach der Wiederaufnahme des Großkönigstitel oder des Mithradatismus, d.h. der ideologischen Bedeutung des Rückverweises auf Mithradates Eupator, gehören in diesen Zusammenhang. ${ }^{15}$

\section{Mithradates von Kios und Mithradates I. Ktistes}

Größere Aufmerksamkeit findet bei Roller die unmittelbare Herkunft des Reichsgründers, wenn auch die kritische Übergangsphase unter Alexander dem Großen praktisch

\footnotetext{
${ }^{13}$ Für Mithradates VI. siehe vor allem Just. Epit. 38,7,1: Se autem, seu nobilitate illis conparetur, clariorem illa conluvie convenarum esse, qui paternos maiores suos a Cyro Darioque, conditoribus Persici regni, maternos a magno Alexandro ac Nicatore Seleuco, conditoribus imperii Macedonici, referat, seu populus illorum conferatur suo, earum se gentium esse, quae non modo Romano imperio sint pares, sed Macedonico quoque obstiterint. Dazu AlidOUST 2020, 327 mit Anm. 1217. Ergänzen könnte man, wie achämenidische Onomastik erst unter Mithradates VI. Eupator florierte (BALLESTEROS PASTOR 2015) oder das Motiv der Sieben auch die spätere Flucht des Mithradates Ktistes allein in der Version Appians (Mithr. 9.28) widerspiegelt. Zur Ahnengalerie des Orontiden Antiochos I. Theos siehe z.B. TOUMANOFF 1963, 277-354; JACOBS 2002; FACELla 2006; STROOTMAN 2016; Versluys 2017. Zu den Ariarathiden von Kappadokien siehe unten Anm. 90; zu den Ariobarzaniden BALLESTEROS PASTOR 2020B.

14 Roller 2020, 14. Eine Abstammung von Dionysos ist allerdings nicht Teil der zeitgenössischen Alexandertradition.

15 Zentrale Arbeiten, die einen ideologischen Iranismus vertreten, welcher in bewusster Opposition zu Griechen und Römern stehe, sind ROSTOVTZEFF 1919 und 1931; vgl. GAJDUKEVIČ 1971; SAPRYKIN 1996; 2002; 2005. Zur kritischen Diskussion des Mithradatismus siehe z.B. HEINEN 2001; 2006; 2008B; COŞKUN UND HEINEN 2004; COŞKUN 2016A; 2020A; 2021A; PRIMO 2009. Achämenidisches Erbe behandeln z.B. auch BALleSteros PASTOR 2011 A (Feindbild von Eupator); 2012 (achämenidische Vorfahren und toponomastische Traditionen); 2015 (Traditionen und Propaganda unter Eupator); 2017 (Pharnakes II. als König der Könige); 2018 (Eupator als König der Könige); 2021 A (Pharnakes II. als Nachkomme der Achämeniden); CANEPA 2020, 106. Zu Titeln und Reichsideologie siehe auch MUCCIOLI 2013, 395-417; ENGELS 2014; 2017 , 41-71; RUSSO 2015; CANEPA 2017; 2020; PAYEN 2020, 289-309; BALlesteros PASTOR 2021 (Pharnakes II. und die achämenidische Tradition); im Erscheinen (Symbolik auf Münzen). Eine Einführung in den Persianismus bieten STROOTMAN UND VERSLUIS 2017, in den von Rostovtzeff geprägten Iranismus MORDVINTSEVA 2021A. Perserkonstrukte in römischer Zeit behandelt ALIDOUST 2020.
} 
unberücksichtigt bleibt. ${ }^{16}$ Bisher ging man meist mit Diodor davon aus, dass ein gewisser Mithradates, Sohn eines Ariobarzanes, von 337/36 bis 302/1 v. Chr. eine kleine Herrschaft in Kios und Umgebung innegehabt hätte, bevor ihn Antigonos Monophthalmos kurz vor der Schlacht von Ipsos töten ließ. ${ }^{17}$ Roller folgt indes der tiefgreifenden Revision von Brian Bosworth und Pat Wheatley. Zu Recht weisen diese darauf hin, dass Diodor Kios lediglich als Schauplatz der Ermordung jenes älteren Mithradates nennt und nicht als seine Residenz. Sein Herrschaftsgebiet habe sich vielmehr über Mysien und Mariandynia an der nordwestanatolischen Schwarzmeerküste erstreckt. All dies ist durchaus möglich, schließt aber Kios als Stammsitz der Familie keineswegs aus. ${ }^{18}$

Ein jüngerer Mithradates, der spätere Ktistes, gilt bei Diodor als sein Sohn, ${ }^{19}$ während Plutarch ihn als Sohn eines Ariobarzanes bezeichnet. In Gefolgschaft des letzteren betrachten ihn Bosworth, Wheatley und Roller als Neffen des Mithradates von Kios. ${ }^{20}$ Dieselben Forscher akzeptieren zudem den von Plutarch und Appian gebotenen Erzählrahmen, nach dem Antigonos jenen Mithradates habe beseitigen wollen, aber Demetrios seinen Freund durch eine geheime Warnung zur Flucht verholfen habe. Die Intervention des Demetrios

\footnotetext{
${ }^{16}$ Dem Thema ist ein halber Satz bei RolLer 2020, 26 gewidmet. Siehe aber CoŞKUN IM ERSCHEINEN F, aufbauend auf HECKEL 2006; 2020, 40-97 und HARRISON 1982; vgl. BOSWORTH UND WHEATLEY 1998, $160 f$. ${ }^{17}$ Diod. 16,89,1 (Archontat des Phrynichos, 337/36 v.Chr.); 16,90,2 (Mithradates, Sohn des Ariobarzanes); 19,40,2; 20,106,1 (Archontat des Nikokles, 302/1 v.Chr.); 20,111,4 (Ermordung des älteren und Flucht des jüngeren Mithradates; je 35 bzw. 36 Herrschaftsjahre; zitiert unten Anm. 19); Plut. Demetr. 4 (Rettung des Sohnes des Ariobarzanes durch Demetrios; zitiert unten Anm. 20); Strab. 12,3,41 (Kimiata; zitiert unten Anm. 23); App. Mithr. 9.27-29. Vgl. Ballesteros PASTOR 2012, 375; 2013, 251-255; D’AgOstini 2016, 87-89; OGDEN 2017, 92-94. Chronologische Ungenauigkeiten bleiben zumeist unkommentiert. So auch bei ROLLER 2020, 26 (und anderswo), der auf 336-302 v.Chr. datiert. AVRAM 2016, 215 weist indes auf die Möglichkeit der Umstellung auf den makedonischen Kalender hin. CoșKUN IN BEGUTACHTUNG A schlägt vor, an der traditionellen Inklusivzählung festzuhalten und betrachtet deswegen die Herrschaftsjahre $35 \mathrm{bzw}$. 36 als vertauscht.

${ }^{18}$ So aber BosWORTH UND WHEATLEY 1998, 155-160; gefolgt z.B. von ROLLER 2018, 717f.; 2020, 25-28.

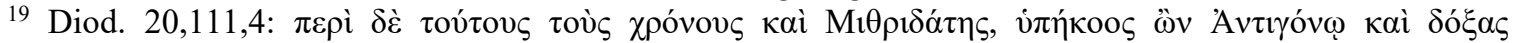

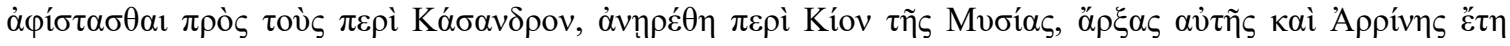

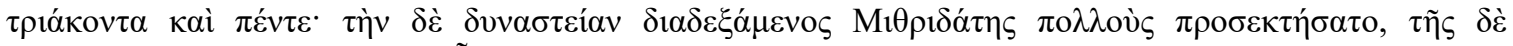

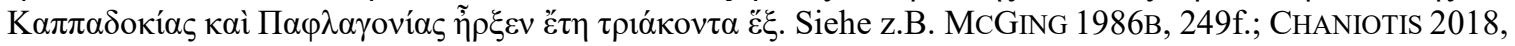
209; CANEPA 2020, 104.

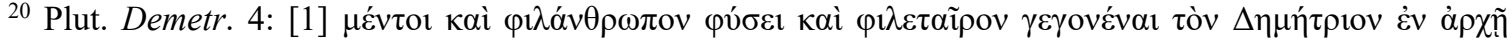

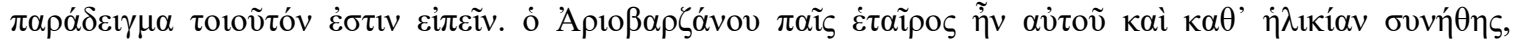

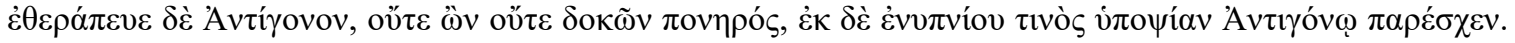

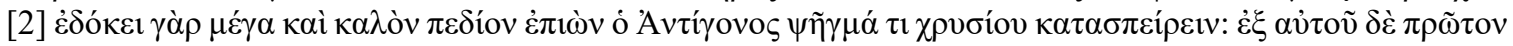

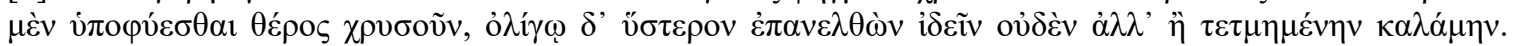

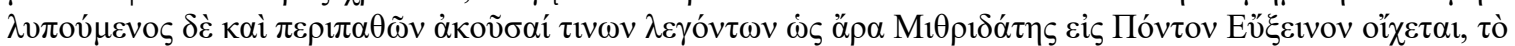

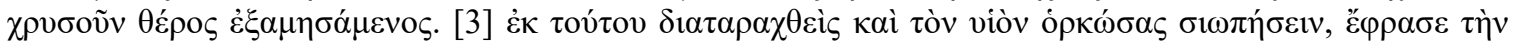

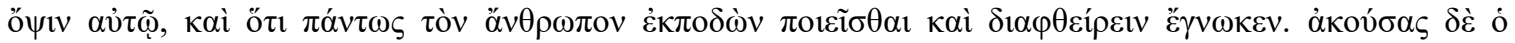

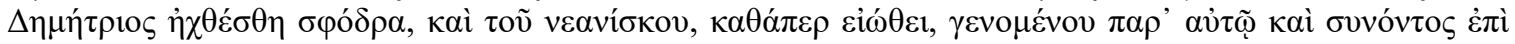

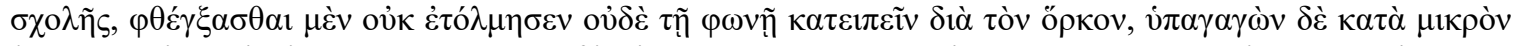

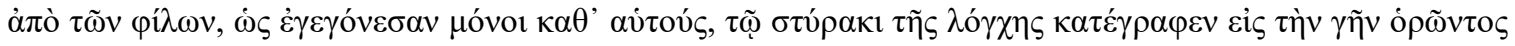
$\alpha$

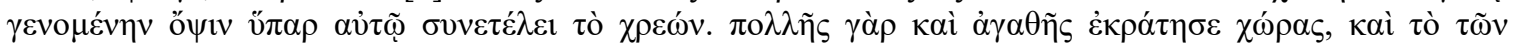

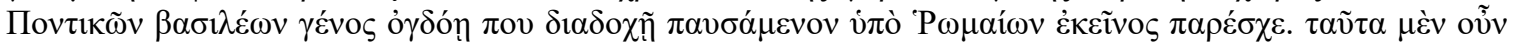

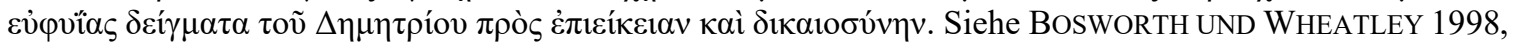
155-160; vgl. D’AGOSTINI 2016, 91; Roller 2020, 26; 33 (die Entscheidung bleibt aber im Stammbaum S. xiii offen).
} 
impliziere, dass die Episode ins Jahr 314 v. Chr. falle und von der späteren Tötung des älteren Mithradates zu trennen sei. ${ }^{21}$ Diese Rekonstruktion überzeugt aber aus mehreren Gründen nicht: Die literarische Ausführung einer Fluchtlegende scheint ein Versatzstück aus der (älteren) Seleukos-Legende zu sein. ${ }^{22}$ Die Einschaltung des Demetrios ist dabei wohl eine apologetische Ergänzung des Hieronymos von Kardia und sollte nicht als chronologischer Anker herangezogen werden. Das Motiv des Antigonos gegen den jüngeren Mithradates ist ganz offensichtlich an den Haaren herbeigezogen und eine Art vaticinium ex eventu (ein Traum, der den künftigen Erfolg des Mithradates Ktistes kundtat). Der fiktive Charakter dieser Version zeigt sich auch darin, dass Antigonos nach dessen Flucht weder gegen denselben in seinem Refugium noch gegen seinen Vater (bzw. Onkel) in Kios vorging. Um nicht allzu sehr gegen den gesunden Menschenverstand zu fabulieren, verlegt die legendenhaft ausgeschmückte Version den Zufluchtsort einfach weiter und unbestimmter vom paphlagonischen Kimiata (Strabon) ${ }^{23}$ nach Kappadokien, obwohl auch dies zum Reich des Antigonos gehörte. Und noch wichtiger: von einem älteren Mithradates von Kios ist gar keine Rede mehr bei Plutarch und Appian. Die Kontamination der beiden Mithradatai ist also ganz transparent.

Rollers Darstellung ist ihrerseits inkonsistent und tut wenig, um den Ansatz von Bosworth und Wheatley zu stärken. Er widerspricht sich selbst, wenn er die Flucht nach Kimiata zuerst auf 302 und sodann unvermittelt auf 314 v. Chr. datiert. Einmal sagt er, Ktistes sei in den 330er Jahren v. Chr. geboren, aber noch im selben Absatz heißt es, „he reached maturity after $310 \mathrm{BC}$;" wieder eine Seite später erscheint Ktistes (wie bei Bosworth und Wheatley) im Kampf an der Seite des Hieronymus gegen Antigonos 315 v. Chr., bevor er begnadigt und ein Begleiter des Demetrios geworden sei; schließlich datiert Roller die Warnung durch Demetrios und die anschließende Flucht noch ins Jahr $314 \mathrm{v}$. Chr. ${ }^{24}$ Die Zeit des Ktistes in Kimiata begreift derselbe Forscher nur als Ausharren in einem Versteck, da seine 36 Herrschaftsjahre erst nach dem Tod des älteren Mithradates begonnen hätten, angeblich nach einer Restitution des Familienterritoriums durch Lysimachos.

\footnotetext{
${ }^{21}$ BOSWORTH UND WHEATLEY 1998, 155-160; ROLLER 2020, 32; WHEATLEY 2020, 216f., nun auch mit dem Vorschlag, dass eine homoerotische Beziehung zwischen Mithradates als dem älteren Partner und Demetrios als dem jüngeren den Ausschlag für die Verfolgung durch Antigonos gegeben haben könnte (219-221), aber die Hinweise sind nicht sehr stark, und wenn auf der Flucht des Demetrios während seiner letzten Kampagne in Anatolien der Fluss Lykos genannt wird, so kann sich dies nicht um den Kelkit Çayı in Pontos handeln, sondern es muss der Çürüksu im karisch-phrygischen Grenzgebiet sein, wie zwingend aus den Einzelheiten des Feldzuges hervorgeht (Milet, Karien, Kilikien, Nordsyrien, Kilikien: Plut. Dem. 46-50). Vgl. GABELKO 2005; 2017. MATYSZAK 2008/15, 1f. vermischt die traditionelle und die revidierte Rekonstruktion miteinander. Nebenbei sei bemerkt, wie umstritten die Chronologie jener Jahre ist. So gibt etwa WHEATLEY 2009 für die Rekonstruktion ausdrücklich Diodor den Vorzug vor Plutarch.

${ }^{22}$ PRIMO 2008; Ogden 2017, 93f. Vgl. D’Agostini 2016, 91, Anm. 33.

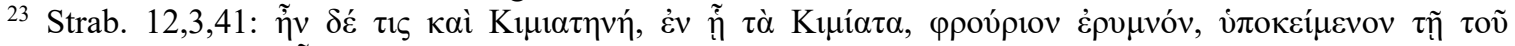

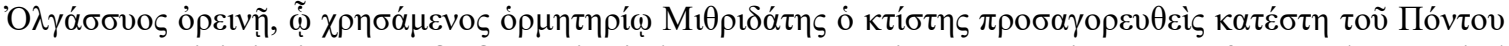

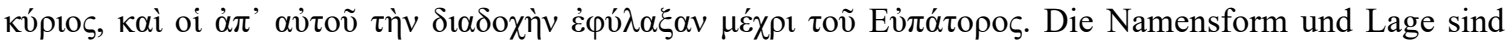
unsicher. Kinista: MAREK 1993, 123f. (mit SEG XXXIII 1097); BOSWORTH UND WHEATLEY 1998, 164; LAFLI UND CHRISTOF 2011, 248. Kimiata: ROLLER 2018, 717; COŞKUN IN BEGUTACHTUNG A. Unentschieden ROLLER 2020, 15.

${ }^{24}$ ROLLER 2020, 15 (302 v.Chr.); 26 (unklar, ob die Flucht vor oder nach Antritt des Erbes der Territorien seines „Onkels“ ergriffen wurde); 30 (Geburt bzw. Volljährigkeit); 31 (315 v.Chr.); 31 (Flucht vor 302 v.Chr.); 32 (Flucht 314 v.Chr.).
} 
Traditionell datiert man die Annahme des Königstitels ins Jahr 281 v. Chr., in dem zuerst Lysimachos im Kampf gegen Seleukos I. fiel und letzterer dann wenig später ermordet wurde. Gemeinhin gilt dies als ein geeigneter Kontext. ${ }^{25}$ Das Jahr basiert vor allem auf der Chronographie des Georg Synkellos. ${ }^{26}$ Etwas großzügig gerechnet dauerte es von da an 218 Jahre bis zum Tod Eupators 63 v. Chr. Allerdings zähle Synkellos jenen gescheiterten König als den zehnten anstatt den achten, wie wir es aus anderen Quellen kennen. ${ }^{27}$ Für Roller ist das Grund genug, das Zeugnis des Synkellos gänzlich zu verwerfen, und das Jahr 297/96 v. Chr. als den Anfang der bithynischen und pontisch-mithradatidischen Ära vorzuschlagen. ${ }^{28}$ So aber schüttet er das Kind mit dem Bade aus. Eine einfache Lösung, die der vorliegenden Überlieferung eher gerecht wird, liegt auf der Hand, da Synkellos doch ausdrücklich auf zwei verschiedene Quellen zurückgreift. Deshalb sind wir keineswegs gezwungen, die beiden Zahlen vollständig zu synchronisieren. Einerseits begann die Königsherrschaft in Pontos wahrscheinlich während der Krise am Hof des Lysimachos nach der Ermordung des Kronprinzen Agathokles 283/82 v. Chr., welche die Ereignisse bis zur Schlacht von Korupedion in Gang setzte, ${ }^{29}$ und sie endete mit der Vertreibung Eupators aus Kleinasien durch Pompeius 66/65 v. Chr. - eine Zeitspanne von insgesamt 218 Jahren, wie sich ohne Rundung bei Inklusivzählung ergibt. Andererseits berücksichtigt die Anzahl der mithradatidischen Könige von Pontos bei Synkellos auch die gescheiterten Versuche des Pharnakes II. (48/47 v. Chr.) und Dareios (39-37 v. Chr.), das Reich ihrer Ahnen zurückzugewinnen. ${ }^{30}$

\section{Die Expansion des Reiches von Mithradates I. bis Mithradates III.}

Nur wenige weitere Ereignisse aus der Zeit des Gründerkönigs lassen sich mit Gewissheit fassen. Dazu zählt vor allem die Eroberung von Amastris, welche während der Samtherrschaft von Mithradates I. Ktistes und seinem Sohn (und Nachfolger) Ariobarzanes erfolgte. Die Kämpfe fallen in die frühen 270er Jahre v. Chr. Sie gehören somit in den zeitlichen und räumlichen Kontext der Neuorientierung in Nordanatolien nach der Ermordung des Seleukos I., wobei vor allem Herakleia einen Führungsanspruch erhob. ${ }^{31}$

\footnotetext{
${ }^{25}$ Roller 2020, 222, Anm. 41. Vgl. MCGing 1986B, 250, Anm. 8; LesChHORN 1993, 81 mit Anm. 22, der Synkellos (siehe unten) freilich irrtümlich unterstellt, die Angabe von 218 Jahren basiere auf einer rein konstruierten Ära; GABELKO UND ZAVOJKIN 2004; GABELKO 2009, 54, Anm. 9.

${ }^{26}$ Synkellos, Chronographia 523 = Apollodor, FGH 244 F 82 = Dionysios, FGH 251 F 5a, mit Übersetzung zitiert bei GABELKO 2009, 47.

${ }^{27}$ Zu Plut. Demetr. 4,4 und App. Mithr. 9,27-29 siehe unten zu Mithradates III. Vgl. PerL 1968, 327: ,erst mit seinem Tod wird nach der Auffassung des Pompeius und der zeitgenössischen Römer die Reihe der pontischen Könige und der Krieg beendet.“

${ }^{28}$ ROLLER 2020, 33-36. Gegen das frühe Datum führt Roller die Loyalität des Ktistes zu dessen Gönner Lysimachos an, aber er lässt offen, warum diese 297 v.Chr. keine Rolle mehr gespielt haben soll. Mehr zur späteren pontischen Ära unten mit Anm. 66.

${ }^{29}$ App. Syr. 64,340f.; auch Paus. 1.10.5; dazu Grainger 1997; 2014; HeINEMANN 2010, 141-155; CARNEY 2013, 33-48; auch OGDEN 2017, 331-333; HANNESTADT 2020, $75 f$.

${ }^{30}$ Siehe oben Anm. 6 zu Pharnakes II. und Dareios. Anders GABELKo 2009, der wenig überzeugend zwei Stadtköniginnen von Prusias ad Mare einbezieht, obwohl nur für eine der Königinnentitel nachgewiesen ist und für keine die Abstammung von Mithradates VI. Eupator; zudem lag Prusias außerhalb des pontischen Territoriums. ROLLER 2020, 194 mit Anm. 10 zitiert zwar Gabelkos Arbeit, geht aber nicht auf sein Argument ein. Unentschieden bleibt BALlesteros PASTOR 2012, 374. Dagegen argumentiert COŞKUN IN BEGUTACHTUNG A.

${ }^{31}$ ROLLER 2020, 36-39. Zur Bibliographie ergänze z.B. HeINEMANN 2010; GALLOTTA 2010; GRAINGER 2014.
} 
Für Roller ist es eine unhinterfragte Tatsache, dass die Ausdehnung des Reiches in die fruchtbare Ebene um Amaseia sowie die Gründung jener neuen Residenzstadt noch unter dem ersten König stattgefunden hätten. Tatsächlich könnten die (heterogenen) literarischen Quellen wegen ihrer vagen Verbindung mit Kappadokien so gedeutet werden, aber dies bleibt in Rollers Argumentation unerwähnt. ${ }^{32}$ Stattdessen verweist er ohne weiteren Kommentar auf eine der Arbeiten von Robert Fleischer zu den Felsgräbern von Amaseia, deren ältestes Ktistes gehören könnte. ${ }^{33}$ Dies ist aber gleich mehrfach spekulativ, da weder die Namen noch Zahl der ,Inhaber' der Gräber bekannt sind. Auch der jeweilige Zeitpunkt ihrer Errichtung ist ungewiss. So tendiert etwa Luis Ballesteros Pastor zur Annexion von Amaseia unter dem Sohn des Ariobarzanes, Mithradates II., während Jakob Højte den Bau der Gräber gar erst unter Mithradates III. beginnen lassen will. ${ }^{34}$ Eine sichere Entscheidung halte ich bei gegebener Quellenlage für unmöglich.

Rasch werden bei Roller die Nachfolger des Gründerkönigs von Ariobarzanes (40) über Mithradates II. (40-49) bis Mithradates III. (50f.) abgehandelt. Die Alleinherrschaft des ersteren lässt Roller 266 v. Chr. beginnen. Allerdings ist es nach Diodor 267/66, wenn nicht (gemäß meiner oben vorgeschlagenen Korrekturen) 268/67 v. Chr. ${ }^{35}$

Natürlich dürfen die Galater in einer Besprechung des Aufstiegs der pontisch-mithradatidischen Herrschaft nicht fehlen, und sie tun es auch bei Roller nicht. Allerdings bedient er sich vor allem des Standardwerks von Stephen Mitchell, das nun aber durch hunderte jüngere Publikationen ergänzt, nuanciert und bisweilen korrigiert wird. ${ }^{36}$ Übersehene Neuerungen betreffen insbesondere die literarische Repräsentation der Galater, ${ }^{37}$ die Zuweisung von Siedlungsplätzen sowie die Deutung politischer Allianzen. Von Einzelbefunden abgesehen verkennt Roller insgesamt die wohl zentrale Bedeutung, welche die tektosagischen Galater für den Aufstieg der Dynastie hatten. Sie ist sehr gut mit dem Anteil vergleichbar, den die Tolistobogier und Trokmer am Erfolg des Nikomedes I. von Bithynien hatten. Dies

\footnotetext{
${ }^{32}$ Hinweise auf die Expansion nach Kappadokien unter Ktistes finden sich bei Diodor (20,111,4, zitiert oben Anm. 19) und Plutarch (Demetr. 4,4, zitiert oben Anm. 20). Nach Appian (Mithr. 9,28) ging die Flucht sogar gleich nach Kappadokien, von wo dann alle weiteren Eroberungen erfolgt seien. Alle drei Varianten könnten aber schlicht aus der Annahme resultieren, dass eine gemeinsame Quelle Amaseia im pontischen Kappadokien als die erste Residenzstadt betrachtete. Hinzu kommt ein terminologisches Problem. Roller selbst erkennt, dass „Kappadokien“ in unseren Quellen oft für Pontos verwendet wurde, und sein Kontext (Amastris) scheint dies sogar auf den paphlagonischen Teil von Pontos auszudehnen $(2020,36 \mathrm{f}$.). Andererseits ist keineswegs sicher, ob Gebiete westlich des Halys in frühhellenistischen Quellen tatsächlich als „Kappadokien“ bezeichnet wurden; genauso gut kann man an Ungenauigkeiten infolge von Kontamination oder Pauschalisierung denken. Ein entsprechendes Beispiel aus dem 1. Jh. v.Chr. bespricht CoșKUN 2021C, $280-282$.

33 Roller 2020, 35f. mit Anm. 45, wo auf FLEISCHER 2009 verwiesen ist (vgl. FlEISCHER 2017); ähnlich z.B. auch MiCHELS 2009, 290.

${ }^{34}$ HøJTE 2009B, 127; BALlesteros PASTOR 2021, $191 \mathrm{f}$.

35 Ohne Begründung operiert Roller mit römischen Jahreszahlen und Exklusivzählung. Zu erwarten ist aber Inklusivzählung, und die Wahrscheinlichkeit, dass Jahreszahlen (35 und 36) miteinander vertauscht wurden, ist hoch, siehe oben Anm. 17.

${ }^{36}$ ROLLER 2020, 37f. und MitCHELL 1993 (zitiert mit Nachdrucksdatum 1995).

37 Roller 2020, 223, Anm. 53 zitiert MitCHELl 2003; ergänze vor allem STROBEL 1994; KISTLER 2009; CoşKUn 2014A.
} 
hätte etwa im weiteren Kontext der Restrukturierung Nordkleinasiens nach der Niederlage des Lysimachos bei Korupedion (281 v. Chr.) erörtert werden können. ${ }^{38}$

Roller datiert die bei Apollonios von Aphrodisias (FGH 740 F 14) bezeugte Abwehr des Ptolemaios II. Philadelphos durch Ariobarzanes und nicht weiter spezifizierte Galater in den Zeitraum 276/66 v. Chr. ${ }^{39}$ Das ist unnötig vage, da die ptolemäischen Offensiven im Ersten Syrischen Krieg 271/70 v. Chr. abgeschlossen waren. Zudem ist es befremdlich, dass Roller jenes Ereignis ins weit entfernte Karien verlegt, wo doch Apollonios den Erfolg der Verteidiger meldet, die angreifenden Ptolemäer Karien aber behielten. Hier passt praktisch gar nichts zusammen. In der Galaterforschung ist eine Lokalisierung an der paphlagonischen Küste allgemein anerkannt, und eine räumliche wie zeitliche Nähe zur oben erwähnten Annexion von Amastris liegt nahe. ${ }^{40}$

Auch für die lange Herrschaftszeit des Mithradates II. stehen die Galater $(41 ; 47)$ neben den Seleukiden im Vordergrund. Besonderes Interesse verdient der Bruderkrieg zwischen Seleukos II. und Antiochos Hierax (43; 48f.). Traditionell datiert man den Konflikt zwischen 242/40 und 236/27 v. Chr., während ich selbst jüngst 246 bis 242 v. Chr. vorgeschlagen habe. Bei Roller fehlt eine Begründung für die von ihm vorgenommene Datierung auf 246-236 v. Chr. Dieser Krieg bietet auch den historischen Kontext für den Beginn dynastischer Eheverbindungen zwischen den Mithradatiden und den Seleukiden sowie die Landnahme um Ankyra durch die tektosagischen Galater unter der Protektion von Pontos. Diese politische Aufwertung von Pontos unter Mithradates II bildet auch den Hintergrund für die mögliche Einbeziehung ${ }^{41}$

\footnotetext{
${ }^{38}$ Tavion wird z.B. von STROBEL UND GERBER 2000 als hethitische Stadt beschrieben, die von den Trokmern im 3. Jh. v.Chr. übernommen worden sei, während der archäologische Befund nicht vor dem 1. Jh. n. Chr. einsetzte. CoşKUN 2011A und 2014 hat aber gezeigt, dass die Trokmer sich erst um 100 v.Chr. östlich des Halys niederließen; siehe auch CoşKUN 2018A zur Verbindung von Eupator mit den Trokmern. Zu den Tektosagen und Ankyra siehe im Folgenden. Zusammenfassend zu den galatischen Siedlungsplätzen siehe jetzt CoşKUN 2021E. Zu den politischen Allianzen siehe CoşKUn 2011A; PAYEN 2020; IM ERSCHEINEN A. Die nützlichste Quellensammlung bietet TOMASCHITZ 2002; einen umfassenden Bericht über die neueste Forschung bietet COŞKUN IM ERSCHEINEN C; siehe vorerst COŞKUN 2013.

${ }^{39}$ ROLLER 2020, 38 mit Anm. 54, mit Apollonios von Aphrodisias, FGH 740 F 14.

${ }^{40}$ CoşKUn 2011 A, 88 mit weiterer Literatur in Anm. 12.

${ }^{41}$ ROLLER 2020, 47 und S. 224, Anm. 30 mit Verweis auf PETKOVIĆ 2009, der seinerseits kaum Interesse an der Chronologie des Krieges hat und nur vage auf ca. 239 bis 238/36 datiert (S. 379). Siehe dagegen CoşKUN 2018 (mit Bibliographie) für 246-242 v.Chr. sowie COŞKUN IN VORBEREITUNG zur Auseinandersetzung mit kritischen Stimmen (z.B. HÄMMERLING 2019, 86-99) und zu weiterführenden Gedanken. Zur Allianz der Tektosagen mit Mithradates I. und II. siehe CoşKUN 2011A und 2018B, 209; 226f.; 250 mit Anm. 135 (Just. 38,5,3). Letzteren Aufsatz zitiert Roller zur Expansion des Pharnakes I. (S. 225, Anm. 31), wozu er aber nichts beiträgt; vielmehr stellt er eine tiefgreifende Revision des Dritten Syrischen Krieges dar, welcher eng mit dem Bruderkrieg sowie dem Eindringen des Mithradates II. und der Tektosagen in Ostphrygien verbunden war. Dies wiederum bleibt bei ROLLER 2020, 43f. gänzlich unberücksichtigt. Weiter überraschend ist es, dass ROLLER 2020, 50 den Angriff eines Mithradates auf Sinope traditionell auf ca. 220 v.Chr., also unter Mithradates III., datiert, obwohl das Hauptargument der wenige Seiten zuvor zitierten Studie von Petković eine Neudatierung unter Mithradates II. auf 228 v.Chr. einfordert. Kritisch gegenüber Petković ist allerdings HÄMMERLING 2019, 139-149, der weitere pontisch-seleukidische Ehen diskutiert. Zur epigamia zwischen beiden Häusern siehe auch D'AGOSTINI 2016, 92-96. Zum Königsbrief aus Kos (IG XII 4.1.213 = PHI 349783 = Rigsby no. 12), der traditionell Spartokos IV. vom Bosporos zugewiesen wird, siehe jetzt den Vorschlag von CoşKUN IN BEGUTACHTUNG B.
} 
Die Schwierigkeit, Mithradates III. historisch zu fassen, legt Roller trefflich dar. Man hätte vielleicht noch klarer aussprechen können, dass manche Forscher seine Existenz mittlerweile sogar ganz leugnen. ${ }^{42}$ Tatsächlich stimme ich Roller aber darin zu, an der traditionellen Zählung festzuhalten. Meines Erachtens sprechen vor allem genealogische Gründe gegen die Annahme, dass Mithradates II. von den 250er Jahren bis ca. 186 v. Chr. und sodann seine Söhne bis in die frühen (Pharnakes I.) bzw. späten (Mithradates IV.) 150er Jahre v. Chr. geherrscht hätten.

Roller führt indes drei andere Gründe für die Beibehaltung der traditionellen Zählung an. Der stärkste ist die von Plutarch (und in Variation auch von Appian) behauptete Zahl von acht Mithradatiden bis zu Eupator - ein Argument, in das auch die oben diskutierte Stelle von Georg Synkellos hätte eingebunden werden können, wäre sie nicht vorschnell verworfen worden. ${ }^{43}$ Als nächstes verweist Roller auf eine Inschrift von Delos, meint aber tatsächlich eine in der Forschung recht kontrovers diskutierte Inschrift vom Kapitol in Rom, welche ihren Stifter Mithradates Philopator Philadelphos, Sohn eines Mithradates, nennt. ${ }^{44}$ Vielleicht ist es die Kopie einer erstmals in den 150er Jahren v. Chr. verfassten Inschrift. In der Frage, ob der Vater des Königs Mithradates II. oder III. war, hilft die Inschrift nicht weiter. ${ }^{45}$ Das dritte vermeintliche Zeugnis sind wiederum die oben erwähnten Felsgräber von Amaseia. Sie sind aber für die hier behandelte Problemstellung ebensowenig belastbar, da wir praktisch nicht wissen, wer in welcher Höhle bestattet war. ${ }^{46}$

\section{Pharnakes I. von Apameia bis zum Pontischen Frieden 179 v. Chr.}

Gemessen an seinen Vorfahren erhält Pharnakes I. eine relativ detaillierte Darstellung (5473). Dies ist angesichts seiner historischen Bedeutung für den Aufstieg von Pontos zu einer

\footnotetext{
42 Roller 2020, 50f. Zur Leugnung siehe z.B. PRIMO 2006; D’AGOSTINI 2016, 95.

${ }^{43}$ Plut. Dem. 4,4 und App. Mith. 9,27-29 sowie Synkellos 523, siehe oben mit Anm. 26f. zu Mithradates I.

${ }^{44}$ Roller 2020, 50 und 224, Anm. 2 mit OGIS 375. Vgl. CIL I ${ }^{2} 730=$ VI $30922=I G U R$ I $9=$ ERRINGTON 2020, 280f., hier zitiert nach PHI 187641:

[rex Metradates Pilopator et Pil]adelpus $\cdot$ regus $\cdot$ Metradati $\cdot \mathrm{f}($ ilius $)$

[populum Romanum amicitiai e]t $\cdot$ societatis $\cdot \operatorname{ergo} \cdot$ quae $\cdot$ iam

[inter ipsum et Romanos? optin] et · legati $\cdot$ coiraverunt

[Nemanes Nemanei f(ilius) Ma]hes Mahei f(ilius)

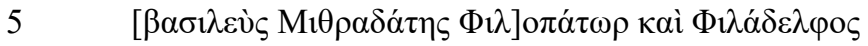

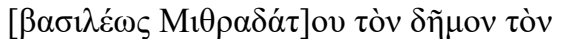

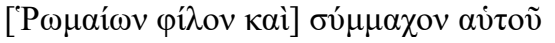

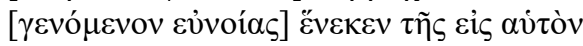

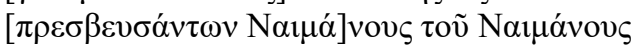

10 [Máov toṽ Máov].

45 Die Stiftung wurde in der Forschung unterschiedlich datiert, doch scheint die erhaltene Version nach dem Kapitolsbrand unter Sulla abgefasst worden zu sein. Gelegentlich wurde der Stifter mit Mithradates V. (Euergetes) oder Mithradates VI. (Eupator) identifiziert, aber seine Gleichsetzung mit Mithradates IV. kann angesichts der Titulatur und Filiation als sicher gelten. Sie ist zuletzt ausgiebig von AVRAM 2016, 220-223 besprochen worden, und ihm ist darin zuzustimmen, dass die Erneuerung der Freundschaft mit Rom nach dem Tod des Pharnakes I. den Anlass für die Gesandtschaft bot. Allerdings argumentiert Avram für ein Datum in den frühen 160er Jahren, da er noch davon ausgeht, dass Pharnakes $170 \mathrm{v}$.Chr. gestorben sei. Eher ist aber mit der Sukzession in den frühen 150er Jahren v.Chr. zu rechnen, siehe unten mit Anm. 63 (IOSPE $\mathrm{I}^{2}$ $402=\mathrm{III}^{3}$ 103) und 70 (Polyb. 27.17). Betreffs des Vaters lernen wir lediglich, dass er Mithradates hieß und er mithin ein Bruder des Pharnakes I. war. Ob ihr Vater Mithradates II. oder III. war, bleibt völlig offen.

${ }^{46}$ Roller 2020, 51 und 224, Anm. 4 mit Verweis auf FLEISCHER 2017, 115. Siehe aber oben mit Anm. 33.
} 
Mittelmacht sehr willkommen. Zutreffend erkennt Roller in der Eroberung Sinopes (184/83 v. Chr.) eine epochale Wende, da diese mit dem Aufbau einer Flotte und der Übernahme weiterer wichtiger Hafenstädte einhergegangen sein müsse. ${ }^{47}$ Allerdings scheint er bei der Formulierung des Satzes „Pontos for the first time moved away from being a state oriented solely in northern Asia Minor" die seit Generationen zum Reich gehörende Hafenstadt Amastris aus den Augen verloren zu haben. Außerdem ist die angenommene Küstenlänge von 400 Meilen eine ziemlich großzügige Schätzung der pontischen Küste unter Pharnakes. Sie übersteigt bei weitem die Summe der Teile, die Roller ihr in seiner Darstellung zugesteht. ${ }^{48}$

Rollers Monographie zur pontischen Reichsgeschichte hätte den geeigneten Rahmen geboten, sich eingehender mit den Konsequenzen des syrisch-römischen Krieges zu beschäftigen, den die Forschung bisher weitgehend aus westanatolischer und seleukidischer Sicht untersucht hat. ${ }^{49}$ Erst jüngst haben die Arbeiten von Germain Payen dazu eingeladen, die Perspektive zu erweitern. ${ }^{50}$ Unbefriedigend ist es jedenfalls, lediglich eine beiläufige Vermutung zu äußern, dass der Friede von Apameia 188 v. Chr. Gebietsverluste für Pharnakes nach sich gezogen haben könnte. ${ }^{51}$ Das ist nirgends belegt und kaum wahrscheinlich, da Pontos schon vor dem Krieg autonom war und sich an den Kämpfen gegen Rom auch nicht beteiligt hatte. Wenige Seiten später argumentiert Roller indes ganz anders, wenn er schreibt, dass erst Prusias I. von Bithynien Pharnakes durch seine Einbindung in die antipergamenische Allianz aktiviert habe. Aber nicht einmal die Existenz eines solchen Bündnisses ist wahrscheinlich. ${ }^{52}$ Gleichfalls überrascht die Deutung der Eroberung Sinopes als zufälliges Ereignis. Dies lässt unberücksichtigt, dass einer seiner Vorfahren bereits

\footnotetext{
${ }^{47}$ Roller 2020, 58f. mit Polyb. 23,9,3; S. 50 datiert er die Eroberung ,in the early 180s BC“.

${ }^{48}$ Wie diese Distanz zu verstehen ist, bleibt unklar, da ROLLER 2020, 58 den eroberten Küstenstreifen mit den Städten Sinope, Amisos, Kotyros und Kerasus beschreibt, wofür er von Stadt zu Stadt die Luftliniendistanz von ca. 180 Meilen angibt, aber das weiter östliche Trapezus ausdrücklich ausnimmt. Auf S. 90 ist erstmals Abonuteichos als Teil des Reiches unter Mithradates V. erwähnt. Mir scheint, dass Roller hier versehentlich die spätere Expansion unter Mithradates VI. Eupator vor Augen hat. Auch die Karte bei PAYEN 2020, 167 scheint Amastris und Abonuteichos dem Territorium des Pharnakes zuzuschlagen, ohne diese Städte aber namentlich zu nennen oder die westliche Ausdehnung des Territoriums zu begründen. Vielleicht ist sie aus dem Feldzug gegen Tios (Tieion) 180 v.Chr. erschlossen.

49 In Ergänzung zur Bibliographie von Roller siehe vor allem MCDONALD 1967; HANSEN 1971; DREYER 2007; ECKSTEIN 2008; GRAINGER 2015A; 2015B; COŞKUN UND ENGELS 2019.

50 PAYEN 2020, 144-185; vgl. 2016; 2021 A; 2021B; IM ERSCHEINEN A; sowie COŞKUN IM ERSCHEINEN D zur genaueren Bedeutung der geopolitischen Bedeutung des Vertragstextes und CoŞKUN IM ERSCHEINEN B zu den Konsequenzen in Nordostanatolien.

${ }^{51}$ Roller 2020, 54 mit S. 225, Anm. 11 verweist auf Polyb. 23.9, aber die pontischen und pergamenischen Gesandtschaften nach Rom finden 183/82 v.Chr. statt und betreffen die Eroberung Sinopes. Zu Apameia siehe unten.

52 Einziges Zeugnis ist Pomp. Trog. Prol. 32: in Asiam bellum ab rege Eumene gestum adversus Gallum Ortiagontem, Pharnacem Ponticum et Prusian, adiuvante Prusian Hannibale Poeno. Der Pontiker ist aber nicht in der Siegesinschrift von Telmessos 184 v.Chr. genannt. Dies gesteht auch PETKOVIĆ 2012, 360f. zu und schließt lediglich, dass Pharnakes nicht an jener Schlacht beteiligt gewesen sei. Aber es fehlen belastbare Hinweise darauf, dass eine frühere Feindschaft mit Eumenes überhaupt bestanden hatte. CHRUBASIK 2013, 108f. erwägt, dass es sich bei Trogus um eine Verwechslung mit dem späteren Krieg von 182-179 v.Chr. handeln könne. Dagegen spricht aber, dass Prusias damals auf pergamenischer Seite kämpfte. Eher vermute ich, dass der Prolog summarisch die Feinde Pergamons zwischen Apameia und dem Pontischen Frieden nennt.
} 
dasselbe versucht hatte und Pharnakes mit seinem bald folgenden Angriff auf Tios (Tieion) 180 v. Chr. sogar noch weiter reichende strategische Ziele demonstrierte. ${ }^{53}$

Die Annexion von Kotyros, Amisos and Kerasus ist für Roller ganz zutreffend eine Konsequenz der Eroberung von Sinope, welches zuvor die Hegemonialmacht entlang des ostpontischen Küstenstreifens gewesen war. Die Annahme der Einverleibung von Trapezus lehnt Roller indes ab, da Strabon den Erwerb erst für Eupator bezeuge. ${ }^{54}$ Hier wäre eine eingehendere Diskussion wünschenswert gewesen. Mir scheint jedenfalls, dass sich Strabons Aussage zur erstmaligen Eroberung durch Eupator nicht auf die Küstenstädte, sondern nur auf das gebirgige Hinterland bezieht. Ähnliche Bedingungen gelten ja auch für Kerasus, das nach allgemeiner Auffassung von Pharnakes unter dem Namen Pharnakeia ausgebaut wurde. Gleichfalls könnte hier die Gründung der Kultstätte des Men Pharnakou in Ameria in der Phanaroia angeführt werden. ${ }^{55}$

Für die Politik des Pharnakes blieb der Blick in Richtung Seleukiden selbst nach Apameia wichtig, so wie sich auch alle anderen Herrscher Kleinasiens dieses potenziellen Machtfaktors bewusst waren. ${ }^{56}$ Deswegen ist es nicht verwunderlich, dass er sich im Krieg gegen Eumenes von Pergamon, Prusias von Bithynien und Ariarathes von Kappadokien nach dem Abfall seiner neuen galatischen Bündner Kassignatos und Gaizatorix 180 v. Chr. an Seleukos IV. wandte. Kurios ist lediglich, dass jener zuerst Hilfe zusagte, dann sein Heer in Marschbereitschaft setzte und schließlich scheinbar unvermittelt seine Meinung änderte. Die Erklärung Diodors, dass jenem in letzter Sekunde die Vertragsbedingungen von Apameia eingefallen seien, ist schwach. Dagegen gehen viele Forscher davon aus, dass der König in letzter Sekunde von römischen Diplomaten gewarnt worden sei. Insbesondere fällt hier der Name des T. Quinctius Flamininus, dessen damalige Tätigkeit zumindest in Kleinasien belegt ist. Dass er auch am Seleukidenhof vorstellig geworden sei, wird in Rollers Darstellung ohne weitere Erörterung zur Tatsache. ${ }^{57}$ Demgegenüber neigen andere Historiker in Ermangelung positiver Belege für eine solche Reise eher dazu, den Abbruch der seleukidischen Kampagne mit ihrem verspäteten Beginn zu erklären. ${ }^{58}$ Noch wahrscheinlicher ist aber eine ganz andere Erklärung: Seleukos hatte bei seiner Zusage ein Desinteresse der Römer an Nord- und Nordostanatolien vorausgesetzt, gelangte nach Unterrichtung über ihren neuerlichen Vorstoß für eine Friedensregelung aber zu einer anderen Risikoeinschätzung. ${ }^{59}$

\footnotetext{
${ }^{53}$ Roller 2020, 54-60. Zu Tios siehe Diod. 29,23; Polyb. 25,2,7. PAYEN 2020, 166 verweist zudem auf den früheren Versuch des Mithradates III., Sinope zu erobern (Polyb. 4,56,1-4,57,1); siehe oben Anm. 41.

${ }^{54}$ Strab. 12,3,17.28, mit ROLLER 2020, 58; 200; vgl. ROLLER 2018A, 709; ähnlich kursorisch PAYEN 2020, 412-414 (Karten); 2021a, 171f. (Erwähnung von Kotyros / Kotyora und Kerasus / Pharnakeia, nicht aber Trapezus).

${ }^{55}$ Strab. 12,3,30f., dazu CoŞKUN IM ERSCHEINEN B.

${ }^{56}$ Siehe PAYEN 2019; vgl. CHRUBASIK 2013. Allgemein zu den Vertragsbedingungen auch ERRINGTON 2020, 83-97.

${ }^{57}$ ROLLER 2020, 60, der seine Deutung wenig überzeugend mit derjenigen Diodors $(29,27)$ harmonisiert. Die Reise des Flamininus nach Antiochien wird z.B. auch von PETKOVIĆ 2012, 362 angeführt.

${ }^{58}$ Siehe GRAINGER 2015B, 6f. und PAYEN 2019, 285f., die sich für letztere Erklärung aussprechen. CHRUBASIK 2013, 109f. lässt den Grund offen, betont aber, dass die Mission des Flamininus nicht in diesem Zusammenhang bezeugt sei.

${ }^{59}$ Siehe COŞKUN IM ERSCHEINEN B.
} 
Vielleicht waren die römischen Gesandten aber auch beeindruckt von Seleukos' grundsätzlicher Interventionsbereitschaft und bauten Pharnakes deswegen goldene Brücken. Anders als Roller das erkennt, ${ }^{60}$ war die letztlich getroffene Friedensregelung viel großzügiger, als es der Pontiker nach einem erneuten Waffengang gegen die pergamenische Allianz hätte erwarten dürfen. Zwar musste er manche binnenländische Gebiete in Paphlagonien und Galatien räumen, aber Sinope und die anderen eroberten griechischen Städte an der Schwarzmeerküste sowie die Phanaroia konnte er behalten, die Tributzahlung war moderat und die Souveränität des mit ihm verbündeten Satrapen Mithradates von Armenien blieb ebenfalls erhalten. ${ }^{61}$ Und abgesichert wurde all dies nicht allein durch einen Friedensvertrag zwischen den Kriegsparteien und weiteren Signatarmächten, sondern zudem durch die offizielle Freundschaft mit Rom - die wohl mächtigste Absicherung gegen eine Wiederaufnahme des Krieges durch die Pergamener oder Kappadoker.

\section{Pontische Diplomatie unter Pharnakes I. nach 179 v. Chr. ${ }^{62}$}

Ein Eckpfeiler für die Rekonstruktion der pontischen Geschichte ist der pontisch-chersonesitische Freundschaftsvertrag aus dem Monat Daisios eines Jahres 157 ,wie Pharnakes

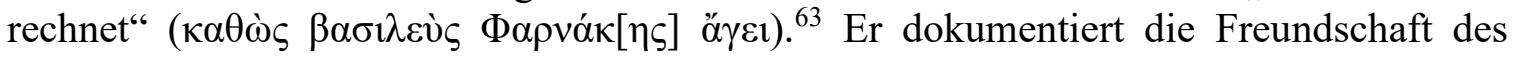
Königs sowohl mit der mächtigen Stadt auf der Krim als auch mit den Römern, und, folgt man wie Roller der Datierung Stanley Bursteins auf April/Mai 155 v. Chr., dann wäre es auch sein letztes Lebenszeichen. ${ }^{64}$ Immerhin weist eine Fußnote auf zwei abweichende Meinungen hin, aber eine Auseinandersetzung mit den Argumenten der neueren Literatur findet nicht statt. So entgeht Roller, dass Heinz Heinen nur indirekt den von Burstein verworfenen Beginn einer ersten pontischen Ära von 336 v. Chr. verteidigt hat. Das Hauptgewicht seiner Argumentation richtet sich vielmehr auf das im Vertrag gespiegelte Beziehungsgeflecht zwischen Pontos, Chersonesos und Rom, für dessen Zustandekommen die intensive Diplomatie gegen Ende des Pontischen Krieges 179 v. Chr. den einzig plausiblen Kontext bildet. Demgegenüber fehlen Spuren eines römischen Engagements im

\footnotetext{
${ }^{60}$ ROLLER 2020, 61: „Pharnakes had gained nothing and had become responsible for a large financial burden, which was still affecting the kingdom nearly twenty years later.“ Ähnlich auch ALLEN 1982, 79. ERRINGTON 2020, 175-180 bleibt ohne Bewertung des Ergebnisses, betont aber zu Recht den Einfluss Roms hinter den Kulissen.

${ }^{61}$ ROLLER 2020, 61; 64, mit weiterer Literatur, besonders PATTERSON 2001. Die (problematische) Theorie von BALLESTEROS PASTOR 2016, dass es sich bei demselben um den künftigen Mithradates IV. von Pontos handle, nimmt Roller nicht zur Kenntnis. Meistens gilt Kleinarmenien als sein Territorium (z.B. CoşKUN 2016B, 851-853; TRAINA 2017). Allerdings ist die Unterscheidung zwischen Klein- und Großarmenien sehr viel später (siehe COŞKUN IM ERSCHEINEN A). An anderer Stelle will ich zeigen, dass der Satrap Mithradates den westarmenischen Zweig der Orontiden fortsetzte, während Artaxias den Osten Armeniens beherrschte. Vgl. vorerst COŞKUN IM ERSCHEINEN B, Anm. 28; siehe auch unten Anm. 93.

${ }^{62}$ Dieser Abschnitt ist eine stark gekürzte Fassung von COŞKUN IM ERSCHEINEN F.

${ }^{63}$ IOSPE $\mathrm{I}^{2} 402$ ed. Latyshev. Die neuesten Texteditionen bieten Makarov $2017=\operatorname{IOSPE} \mathrm{III}^{3} 103$ und ERRINGTON 2020, 180-184.

${ }^{64}$ Roller 2020, 62 und 65, mit Verweis auf BURSTEIN 1980 und HøJTE 2005. Vgl. MCGing 1986B, 254; STOLBA 2005, 161f.; MÜLLER 2010, 93-95; 391-393; auch LESCHHORN 1993, 78 (der allerdings auf 157 v.Chr. datiert) und 82: „Nicht ein historisches Ereignis in der eigenen Geschichte führte also zur Verwendung der ersten Ära in Pontos, sondern die in der Praxis am besten verwendbare Zeitrechnung“" (sc. der Seleukiden).
} 
Schwarzmeerraum in den nachfolgenden Jahrzehnten. Damit hat Heinen die Mehrheit der jüngeren Forschung hinter sich vereinigt, und wohl auch zu Recht. ${ }^{65}$

Fragen betreffs des Ärenbeginns sind aber auf beiden Seiten offengeblieben. Ab dem Jahr 96/95 v. Chr. ist sicher die von Mithradates VI. Eupator verwendete Ära belegt, welche wie auch die bithynische - ihren Anfang 297/96 v. Chr. nimmt. Freilich ist es möglich, dass diese spätere Ära schon zweimal unter Mithradates V. (Jahr 161 und $173=137 / 36$ ? und 125/24? v. Chr.) bezeugt ist. Für den oben genannten Vertrag des Pharnakes mit Chersonesos beginnt diese offenbar zu spät, da derselbe schon lange vor 140 v. Chr. gestorben war. ${ }^{66}$

Die Inanspruchnahme der seleukidischen Zeitrechnung nach dem Rückzug dieser Dynastie aus Kleinasien ist viel problematischer, als Burstein es eingesteht. Es soll an dieser Stelle reichen, darauf hinzuweisen, dass Wolfgang Leschhorn in der detailliertesten Besprechung von möglichen Parallelen regelmäßig große Ungewissheit einräumt, bevor er sich dann aber wegen des vermeintlich erwiesenen Falls von Pontos dennoch für die Seleukidenära ausspricht. ${ }^{67}$ Ein offenbarer Zirkelschluss! Unbeachtet bleibt ferner die Tatsache, dass das Mithradatidenreich den Seleukiden niemals unterworfen war.

Auf der anderen Seite bleiben Leschhorns rechnerische Vorbehalte gegen eine Kios-Ära bestehen: Der Herrschaftsantritt des Mithradates von Kios während des Archontats des Phrynichos 337/36 v. Chr. liegt ein Jahr zu spät, um den Bündnisvertrag auf 179 v. Chr. zu datieren; er wäre dann schon im Jahr vor der Beschwörung des Pontischen Friedens abgeschlossen worden. Alexandru Avram hat nun einen möglichen Ausweg gezeigt, indem er Indizien für eine ähnliche Ära von Sinope vorgelegt hat, die Pharnakes genutzt haben könnte und einen angemessenen zeitlichen Spielraum gewährt. Wie ich aber an anderer Stelle darlegen werde, halte ich es für die plausibelste Lösung, dass die Bestätigung des Mithradates von Kios durch Alexander nach der Schlacht am Granikos im Frühjahr 334 v. Chr. zum Bezugspunkt der ersten pontischen Ära gewählt wurde. Der Vertrag mit Chersonesos wäre dann etwa im Mai 178 v. Chr. beschworen worden. ${ }^{68}$ Sicher können wir zum

\footnotetext{
${ }^{65}$ Heinen 2005 (vgl. Roller 2020, 226, Anm. 58); PATON, WALBANK UND HABICHT 2012, 468f.; COJOCARU 2014, 53, Anm. 55; ERRINGTON 2014 (zu Unrecht von ROLLER 2020, 226, Anm. 60 in Anspruch genommen) sowie jetzt 2020, 180-184; AVRAM 2016; PAYEN 2019, 287; 2020, 169-187; 2021a, 166; 176-178; zögerlich und ohne Argumentation auch MAKAROV 2017. Zur älteren Forschung siehe ferner PERL 1968, 301f., der vorsichtig zu einem Ärenbeginn von 336 v.Chr. neigt. Unentschieden bleibt BALLESTEROS PASTOR 2012, 372, Anm. 14.

${ }^{66}$ Anders freilich die in der vorletzten Anm. genannten Forscher, welche die Seleukidenära ansetzen. So auch ROLLER 2020, 90, obwohl der in seiner Bibliographie gelistete Titel von DE CALLATAŸ 2009 (78f.) gewichtige Gründe dafür anführt, dass die ärendatierte Münze des Mithradates V. eine Fälschung ist. Allgemein zu den in Pontos verwendeten Ären siehe LESCHHORN 1993, 78-96; auch 44-66 zum Bosporanischen Reich, in dem die mithradatidische Ära fortgeführt wurde. Vgl. PERL 1968, 301f.; BICKERMAN 1980, 72.

${ }^{67}$ LESCHHORN 1993, 22-43. Die umfassendste Behandlung der seleukidischen Ära bietet jetzt KOSMIN 2018, der ein sehr optimistisches Bild von ihrer Verbreitung zeichnet, aber nicht auf Pontos eingeht oder andere Beispiele aus Kleinasien für die Zeit nach Apameia bespricht. Ebenso unberücksichtigt ist Pontos bei STROOTMAN 2015.

${ }^{68}$ LESCHHORN 1993, 80; AVRAM 2016; CoșKUN IM ERSCHEINEN F.
} 
jetzigen Zeitpunkt allein dahingehend sein, dass diese Diskussion noch lange anhalten wird. ${ }^{69}$

Roller hätte immerhin damit punkten können, dass ein weiteres Element der Frühdatierung des pontisch-chersonesitischen Vertrags auf Sand gebaut war: die Annahme, dass Pharnakes 170 v. Chr. gestorben sei. Hierfür hat man sich zu Unrecht auf eine Polybios-Stelle berufen, die nichts dergleichen belegt. ${ }^{70}$ Nicht einmal die Tatsache, dass es eine entsprechende Kontroverse gibt, erfährt Rollers Leserschaft, und er vergisst dies gelegentlich selbst, wenn seine Darstellung inkonsequenter Weise wieder die traditionelle Frühdatierung voraussetzt. ${ }^{71}$ Die entsprechende Diskussion biographischer Daten hätte außerdem mit der Besprechung einer Inschrift von Delos verbunden werden können, in der die Athener den König und seine Braut Nysa als Euergeten ehren. ${ }^{72}$ Obwohl die neuere Forschung nun zumeist um eine Generation früher datiert, gehört die Ehrung recht sicher ins Jahr 160/59 v. Chr., wofür sich auch Roller entscheidet. ${ }^{73}$ Die Inschrift belegt nicht nur ein erfolgreiches Interagieren des Pharnakes mit den Athenern, sondern auch eine erneute Annäherung an die Seleukiden unter Demetrios I. (162-150 v. Chr.). ${ }^{74}$

Ebenso in den Kontext der pontischen Diplomatie unter Pharnakes fällt eine Behauptung, die Justin dem Mithradates Eupator in den Mund legt, dass nämlich sein Großvater zum Nachfolger des Eumenes in Pergamon designiert worden sei. ${ }^{75}$ Roller hält dies unter Berufung auf einen Aufsatz von Ballesteros Pastor für historisch, übersieht aber, dass derselbe Autor seine frühere Argumentation schon längst widerrufen hat. Sein ehemaliges positives Urteil hatte auf der Annahme basiert, dass Eumenes II. in den 160er Jahren v. Chr. keinen eigenen Erben besessen hätte. ${ }^{76}$ Aber selbst das damalige Fehlen eines Sohnes hätte eine

${ }^{69}$ GABELKO 2017 setzt den Beginn der Zeitrechnung mit der Flucht des Mithradates Ktistes ca. 314 v.Chr. an, wobei er der (oben abgelehnten) Chronologie von BOSWORTH UND WHEATLEY 1998 folgt; vgl. GABELKO 2005.

${ }^{70}$ Polyb. 27.17. Für die Frühdatierung siehe die Verweise oben in Anm. 44f. (OGIS 375) und 65 (IOSPE $\mathrm{I}^{2}$ $402=\mathrm{III}^{3}$ 103). Sie ist aber durch die delische Inschrift (OGIS 771, siehe unten Anm. 72) widerlegt.

71 Z.B. geht ROLLER 2020, 74 entgegen seiner eigenen Datierung davon aus, dass Pharnakes Anfang der 150 er Jahre v.Chr., also vor Vertragsabschluss, gestorben sei.

72 BCH 29, 1905, no. 61 ed. F. Durrbach = IDelos 1497 bis $=$ OGIS 771 ed. W. Dittenberger (171/70 v.Chr.) = PHI 63933 = GHIȚA 2011 (160/59 v.Chr.).

73 ROLLER 2020, 23f. setzt noch die Frühdatierung voraus, allein mit dem Verweis (S. 221, Anm. 59) auf HABICHT 1997, 226f. Demgegenüber setzt er S. 54 (anstelle eines Belegs findet sich S. 225, Anm. 225 ein offengebliebener Querverweis) und S. 67f. (ohne Literatur) 160/59 v.Chr. an. Dass die Datierung umstritten ist, spricht Roller erst auf S. 68f. an, mit Verweis auf die Gegenpositionen von HABICHT 1997 und GHIŢA 2011. Für 160/59 v.Chr. siehe auch WALBANK 1979, Bd. 3, 318; ALLEN 1982, 117; MCGING 1986B, 254; LESCHHORN 1993, 79; EHLING 2008, 140; BALLESTEROS PASTOR 2013, 248; STROOTMAN 2019, 190. Für die Frühdatierung auf 196 v.Chr. siehe hingegen TRACY 1992, 307-313 (kritisch hierzu aber z.B. TRAILL 1993); HABICHT 1997, 226f; HøJTE 2005, 142f.; HeInen 2005, 40; BALlesteros PASTOR 2007/10; MiCHELS 2009, 89; MuCCIOLI 2013, 141 mit Anm. 570; AVRAM 2016, 216; ERRINGTON 2020, 182f. (im Widerspruch hierzu wird aber S. 145 der Herrschaftsantritt des Pharnakes erst auf ca. 185 v.Chr. datiert).

${ }^{74}$ Zur historischen Einordnung siehe - neben den Verweisen oben in Anm. 73 - auch HÄMMERLING 2019, 54; 59; COŞKUN 2021D und IM ERSCHEINEN B.

75 Just. 38,6,2: Sic et auum suum Pharnacen per cognitionum arbitria succidaneum regi Pergameno Eumeni datum.

76 Roller 2020, 67 mit Anm. 71-73 und Ballesteros Pastor 2000/2001 mit Polyb. 30,2,6; contra Ballesteros PAStOR 2013, 251-255. HANSEN 1971; AlLEN 1982, 81 und PAYEN 2020 ignorieren die JustinStelle. 
Designation des Pharnakes nicht plausibel gemacht, da ja Attalos (II.) schon lange die rechte Hand seines Bruders war und auch als sein Erbe gehandelt wurde. Ein angemessenes Verständnis der Justin-Stelle muss den rhetorischen Kontext genauer berücksichtigen. Vielleicht handelt es sich bei dieser ganz offensichtlichen Lüge um eine Legitimationsstrategie Eupators, der während seines ersten Krieges gegen Rom Pergamon besetzt hielt und dort auch residierte. ${ }^{77}$

\section{Mithradates IV. Philopator Philadelphos und seine Gattin Laodike}

In Rollers fünftem Kapitel erhalten die beiden folgenden Könige zusammen weniger Text als Pharnakes, was wohl ihrer schlechteren Dokumentation geschuldet ist. ${ }^{78}$ Die Nachfolge des Mithradates IV. auf seinen Bruder wird zurecht mit dem jungen Alter des Mithradates V. erklärt. Dies hätte als weiteres Argument für die Spätdatierung der Heirat von Pharnakes und Nysa dienen können. Treffend ist auch der Hinweis, dass die erstmalige Annahme von Herrschaftstiteln durch Mithradates IV. Philopator Philadelphos die fortschreitende Hellenisierung der Mithradatiden impliziert. Allerdings ist die Behauptung, dass der Titel Philadelphos zwangsläufig eine inzestuöse Ehe mit Laodike voraussetze, etwas voreilig. ${ }^{79}$ Wie nämlich bei seinem Zeitgenossen Attalos II. kann dieses Epitheton auch schlicht die liebevolle Treue zu seinem Bruder bezeichnen. Dies liegt umso näher, als er seinen Bruder beerbt hatte und zugleich seinen Neffen zum Nachfolger designierte, so wie es auch bei Attalos II. / III. und Antigonos III. Doson / Philipp V. der Fall war. ${ }^{80}$

Es mutet sonderbar an, die Entscheidung für Inzest als die beste Option des Mithradates zu bezeichnen, während eine Ehe mit einer Tochter des unter turbulenten Bedingungen an die Macht gekommenen Demetrios I. nicht opportun gewesen sei. Dies ist gewiss eine Fehleinschätzung. Schon Pharnakes hatte eine Braut aus der Familie des Demetrios geheiratet, und zwar noch bevor dieser vom römischen Senat 159 v. Chr. als rechtmäßiger König anerkannt wurde. ${ }^{81}$ Zudem ehelichte Mithradates V. wenig später eine weitere Seleukidin, nun wahrscheinlich eine Tochter des Demetrios I. ${ }^{82}$ Außerdem stellt sich die Frage, ob die Ehe mit der Tochter eines anderen Königs oder Stammesfürsten politisch nicht von größerem Vorteil gewesen wäre als Endogamie. Die inzestuöse Heiratspraxis der Ptolemäer war der Sonderfall, nicht die Regel, und somit schwerlich auf die Pontiker übertragbar. Auch die Annahme, dass die Seleukiden häufiger Geschwisterehen geschlossen hätten, wäre erst noch zu beweisen. Die meisten Belege beziehen sich tatsächlich auf rituelle Rollen, in

\footnotetext{
${ }^{77}$ Siehe CoŞKUn IM ERSCHEINEN B zu Eupators Rhetorik und CoșKUn 2011B zu den Attaliden nach Polyb. 30,2 .

${ }^{78}$ ROLLER 2020, 74-93, allerdings mit mehreren Illustrationen und (zu) viel Raum für Nebenschauplätze wie Karthago, Makedonien und das Attalidenreich.

${ }^{79}$ ROLLER 2020, 74-76. Zur Hellenisierung siehe auch OLSHAUSEN 1974; MiCHELS 2009.

${ }^{80}$ Beide Möglichkeiten führt MUCCIOLI 2013, 212f. mit weiteren Quellen und Überlegungen aus.

${ }^{81}$ Siehe COŞKUN 2021D. Zu Demetrios I. siehe auch GRAINGER 2015B, 36-65. Immerhin hätte sich Roller auch auf HÄMMERLING 2019, bes. 30f. berufen können, sowohl für die Verklärung der Geschwisterehe als auch für Mithradates IV. Obwohl ich auch mit Hämmerlings genealogischen Rekonstruktionen nicht vollständig übereinstimme, bietet er nun die umfassendste Zusammenstellung und Analyse inzestuöser Herrscherehen in der Antike.

82 Über die Mutter Eupators ist aber kaum etwas bekannt, nicht einmal ihr Name, wenngleich sie wohl auch Laodike genannt worden sein dürfte; siehe ROLLER 2020, 92. Beredt ist das Schweigen über sie in Hämmerlings Kapitel zu den pontisch-seleukidischen Eheverbindungen (2019, 139-149).
} 
denen das Herrscherpaar die Ehe von Zeus und Hera oder ihren orientalischen Entsprechungen repräsentierte. ${ }^{83}$

Des Weiteren ist die Frage nach dem Alter und dem üblichen Wunsch, Nachkommen zu zeugen, zu berücksichtigen: Mit einer vielleicht 50 Jahre alten Schwester wäre auf diesem Gebiet nicht viel zu erreichen gewesen. Andererseits könnte dies gerade im vorliegenden Fall kein Hinderungsgrund gewesen sein, damit eine Konkurrenzsituation für Mithradates V. verhindert würde. ${ }^{84}$ Die Altersfrage hätte auch in Anbetracht der zwei Abbildungen von Laodike gestellt werden können: Ihr Lebensalter auf dem Doppelportrait einer undatierten, aber eindeutig beschrifteten Münze vermag ich nicht zu schätzen. ${ }^{85}$ Anders verhält es sich bei dem makellosen Damenportrait eines Intaglio von Amisos. ${ }^{86}$ Warum es sich hierbei um Laodike handeln soll, dazu noch nach dem Tod ihres Mannes, erschließt sich mir nicht, umso weniger, als die dargestellte junge Frau kein Diadem, sondern einen Haarreifen trägt und bestenfalls in ihren frühen 30er Jahren erscheint. Spätestens dieser Umstand hätte genealogische Fragen aufwerfen sollen.

Verwirrend ist auch die Nummerierung der Laodikai. Die Gattin des Mithradates II. ist bei Roller nicht Laodike I., sondern Laodike IV.; deren Töchter sind bereits Laodike X. und XI., während die angebliche Schwestergattin des Mithradates IV. keine Ziffer mehr erhält. Eine Kontaminierung dynastischer Zählungen von Seleukiden und Mithradatiden erklärt diesen Befund nur teilweise. ${ }^{87}$ Im Übrigen hätte die prominente Rolle der Gattin des Mithradates IV. Anlass dazu geben können, die Identität der Namenspatronin von Laodikeia Pontika (Ladik, südlich von Amisos / Samsun) zu erörtern. Jedoch ist diese Stadt bei Roller nirgends erwähnt, nicht einmal auf den beiden Karten zu Pontos. In der Forschung werden praktisch alle Könige von Mithradates II. bis VI. als mögliche Gründer diskutiert. ${ }^{88}$

Nur ein kriegerisches Ereignis wird gemeinhin mit Mithradates IV. verbunden, und zwar die Unterstützung des Attalos II. im Krieg gegen Prusias II. zusammen mit Ariarathes V. im Jahre 155/54 v. Chr. ${ }^{89}$ Diese Allianz wurde in den Augen Rollers dadurch motiviert, dass die Könige von Pontos und Kappadokien durch ihren gemeinsamen Vorfahren

\footnotetext{
${ }^{83}$ Kultisch-rituelle Repräsentationsformen von Geschwisterehen sind bei HÄMMERLING 2019, bes. 33 nur beiläufig und als Konsequenz inzestuöser Ehen genannt, nicht aber als mögliche Ursache der Konstruktion geschwisterlicher Rollen für königliche Paare, die sicher nicht inzestuös waren. Die seleukidische Tradition lässt sich schon auf Seleukos I. und Apama (Liv. 38.13: Apama sorore Seleuci regis) sowie Antiochos I. und Stratonike (I.Ilion 32,22) zurückverfolgen; siehe JONES 1993, 81-88 mit zahlreichen weiteren Belegen, vor allem für Antiochos III. und Laodike III. Dies wirft erhebliche Fragen für alle anderen Fälle auf.

${ }^{84}$ Allerdings argumentiert Roller keineswegs mit einer bewussten Enthaltsamkeit.

${ }^{85}$ Roller 2020, 75, Fig. 5.2, mit Verweis auf Numismatica Ars Classica NAC AG, Auction 59, Lot 658. Vgl. DE CALlataŸ 2009, 77f.; MiCHELS 2009, 299, nach dem „,die Geschwisterehe auf Münzen propagiert wurde".

${ }^{86}$ ROLLER 2020, 79, Fig. 5.4, mit Verweis auf Metropolitan Museum 42.11.26.

${ }^{87}$ ROLLER 2020, xiii (Stemma); 43f.; 46 sowie 223, Anm. 7, mit irreführendem Verweis auf GRAINGER 1997, der aber klar zwischen einer systematisch-prosopographischen (arabischen) und einer dynastischen (römischen) Zählung unterscheidet.

${ }^{88}$ Zur Forschung siehe MiCHELS 2009, 298-300.

${ }^{89}$ ROLLER 2020, 76, mit Polyb. 33.12.1 etwa zum Jahr 155/54 v.Chr. Ebenso MCGING 1986B, 254; auch noch PAYEN 2021A, 169.
} 
Mithradates II. verwandt gewesen seien. Tatsächlich hatte Blutsverwandtschaft in hellenistischen Herrscherhäusern, zumal in so dünner Konzentration, aber keinerlei politische Relevanz. ${ }^{90}$ Jedoch hat Payen jüngst gezeigt, dass der von Polybios genannte Mithradates unmöglich ein König von Pontos gewesen sein kann, da sein Kontingent unter der Führung des Prinzen Demetrios, eines Sohnes des Ariarathes V., in Bithynien eintraf und auch kommandiert wurde. ${ }^{91}$ Die Identität jenes Bündners der Kappadoker bleibt freilich umstritten. ${ }^{92}$

Diese vermeintliche Allianz zwischen Kappadokien und Pontos zieht Roller weiterhin dazu heran, um Mithradates IV. als Innovator darzustellen. Unter dem Eindruck des Schicksals, das Perseus im Dritten Makedonischen Krieg (171-168 v. Chr.) erlitten hatte, habe Mithradates IV. einen konzilianteren Weg mit Rom eingeschlagen. Roller hat im vorliegenden Teil seiner Ausführungen schlicht vergessen, dass der oben besprochene Vertrag mit Chersonesos auch ein Freundschaftsverhältnis zwischen Pharnakes und Rom bezeugt, wodurch die gesamte Argumentation hinfällig wird..$^{93}$ Das wenige, das wir von Mithradates IV. wissen - seine in der oben angeführten kapitolinischen Inschrift bezeugte Erneuerung der Freundschaft mit Rom und seine Herrschaftstitulatur sowie die treue Gewährleistung der Sukzession seines Neffen -, belegt eine dezidierte Kontinuität in der politischen Ausrichtung unter dem Nachfolger des Pharnakes.

Mit dem Ausscheiden des bereits erwähnten Zeugnisses des Polybios verlieren wir schließlich einen der wenigen chronologischen Anker der Herrschaftszeit des Mithradates IV. Praktisch könnte er fast die gesamten 150er Jahre an der Macht gewesen sein oder vielleicht auch nur eine kurze Zeit um 150 v. Chr., bevor sein Neffe erstmals während des Dritten Punischen Krieges belegt ist.

\footnotetext{
${ }^{90}$ Freilich gab es enge dynastische Verbindungen zwischen den Herrscherhäusern auch schon vor Mithradates VI. Eupator, dessen Neffe und Sohn als Ariarathes VIII. bzw. IX. den kappadokischen Thron innehatten. Siehe BAllesteros PASTOR 2008; 2016; 2020A; 2020B; HÄMMERLING 2019, 149-155; CANEPA 2020, 107-109.

91 Polyb. 33,12,1 mit PAYEN 2020, 225, der auch auf die unpraktische geographische Implikation hinweist, dass pontische Kontingente kaum über Zentralkappadokien nach Pergamon oder Bithynien entsandt worden wären.

92 PAYEN 2020, 225 hält ihn für den oben in Anm. 61 besprochenen Satrapen.

${ }^{93}$ ROLLER 2020, 76f. Hier zeigen sich wohl Spuren einer früheren Fassung aus der Zeit, als Roller noch an eine Heirat mit Nysa ca. 196 v.Chr. und einen Tod des Pharnakes um 170 v.Chr. glaubte. Vielleicht ist Roller aber auch durch das Zeugnis Appians (Mithr. 10,30) irregeleitet worden, nach dem Mithradates (sc. V., nicht IV.) als erster Pontiker Freundschaft mit Rom geschlossen habe. Dies ist ein offensichtlicher Fehler Appians (so auch ERRINGTON 2020, 323), sei es, dass er durch das Fehlen der früheren (uns nur epigraphisch bekannten) Verträge im originalen Polybios-Text bedingt ist, oder sei es, dass die Quelle Appians vielmehr eine erstmalig effektive Waffenallianz zwischen Rom und Pontos zum Ausdruck gebracht haben mag. Nebenbei sei erwähnt, dass ERRINGTON 2020, 281 nicht von einer Erneuerung der Freundschaft mit Rom durch Mithradates IV. ausgeht, sondern ihn bereits als Mitsignatar des oben besprochenen Freundschaftsvertrages von 179 v.Chr. ansieht. Errington stützt seine Argumentation auf die wenig wahrscheinliche Annahme, dass derselbe Mithradates (IV.) auch neben Pharnakes im Pontischen Frieden genannt sei, doch dürfte jener vielmehr mit dem (orontidischen) Satrapen Mithradates von Armenien identisch sein; siehe Polyb. 25,2,3.11 sowie weiteres zur Diskussion oben in Anm. 61.
} 


\section{Mithradates $V$. Euergetes}

Das genaue Datum der Sukzession des Mithradates V. ist unbekannt, aber da er kaum vor 160 v. Chr. geboren sein kann, wäre es durchaus denkbar, dass seine Tante Laodike, die Witwe des Mithradates IV., übergangsweise eine aktive Rolle am pontischen Hof einnahm. Vielleicht ist sie sogar schon zu Lebzeiten ihres Gatten hierauf vorbereitet worden. ${ }^{94} \mathrm{Be}-$ zeugt ist ihr Neffe erstmals als König während des letzten römischen Krieges mit Karthago (149-146 v. Chr.), in dem er die Römer mit Schiffen und Truppen unterstützt haben soll. Da unsere Quellen aber keine persönliche Teilnahme an der Kampagne belegt, sagen sie noch nichts Genaues über sein damaliges Lebensalter aus. ${ }^{95}$

Erst im Kontext des Aristonikoskrieges (133-129 v. Chr.) erfahren wir mehr über Euergetes aus unseren Quellen, aber nicht wirklich von Roller. ${ }^{96}$ Die Belohnung des Königs mit Großphrygien erwähnt er nur kurz, wobei lediglich ein beiläufiger Nebensatz von Bestechung spricht. ${ }^{97}$ Hier hätte darauf hingewiesen werden können, dass derlei Vorwürfe später in den Verhandlungen zwischen Sulla und Mithradates Eupator vorgebracht wurden, nachdem die Römer das Territorium nach dem Tod des Euergetes wieder eingezogen hatten. Der eigentliche Streitpunkt war, ob Euergetes einen Kaufpreis bezahlt oder mit Geld nachgeholfen hatte, dass Aquillius ihm das Land schenkte. Weitere Fragen hätten den allein von Justin formulierten Anspruch betreffen können, dass dasselbe Gebiet schon von Seleukos II. dem Mithradates II. als Mitgift für Laodike, die Schwester des Seleukos II., überlassen worden sei. Außerdem wäre von Interesse gewesen zu klären, inwiefern der römisch-pontische Territorialhandel die dort ansässigen Galater betraf. ${ }^{98}$

Immerhin spricht Roller manche dieser Probleme an, aber erst nach weiteren Abschweifungen zu den langfristigen Ereignissen im Mittelmeerraum (85f.), zum Titel Euergetes (86f.), ${ }^{99}$ und erneut zur Beteiligung am Krieg gegen Aristonikos (87). Bisweilen hat man den Eindruck, dass hier Textfragmente verschiedener Zeit ohne Rücksicht auf Stringenz zusammengefügt wurden. Endlich nimmt Roller die verschiedenen Expansionsbestrebungen des Mithradates V. nach Bithynien, Kappadokien und Galatien doch noch in den Blick, und zwar vor allem durch die Linse der später unter Mithradates VI. entbrannten Streitthemen. Die Zahl der Ungenauigkeiten ist groß, die der rezipierten neueren Literatur gering,

\footnotetext{
${ }^{94}$ Allerdings sollte sich diese im Grunde plausible Ansicht nicht mehr auf eine Münze berufen, die allein das Portrait der Laodike Epiphanes kai Philadelphos zeigt, da es sich wohl um eine Fälschung handelt: DE CALLATAŸ 2009, 83. Rollers genaue Position hierzu bleibt unklar: 2020, 78f.

95 Roller 2020, 77-79, mit App. Mithr. 10,30. Verwirrend ist, dass Roller 2020, 80 dasselbe Zeugnis für Mithradates IV. in Anspruch nimmt (siehe oben Anm. 93). Weitere Quellen (App. Mithr. 12,38; 56,228) sind bei ERRINGTON 2020, 322-324 zitiert.

96 ROLLER 2020, 81-84 und 228f. breitet die Vorgeschichte so aus, als ob seine Leserschaft noch nichts von den Attaliden erfahren hätte, und wiederum unter Verzicht auf Standardwerke oder neuere Literatur. Z.B. fehlen HANSEN 1971; THONEMANN 2013. Angesprochene Fragen zur Erbfolge oder zur Authentizität des Testaments hätten zudem von DREYER 2005 und COŞKUN 2011B profitieren können.

${ }^{97}$ ROLLER 2020, 84: ,,although bribery of Aquillius was suspected“, mit Just. 37.1.1f. und App. Mithr. 57.237.

98 App. Mithr. 57,237; Just. 38,5,3; dazu die Diskussionen bei PETKOVIĆ 2009, 383; BALLESTEROS PASTOR 2013, 85-87; 240f.; COŞKUN 2018, 209; 226f.; 250 mit Anm. 135; HÄMMERLING 2019, 139-142.

99 Vgl. MuCCIOLI 2013, bes. 192.
} 
und ein klares Bild von der Politik des Königs ist nicht zu erkennen. ${ }^{100}$ Das Kapitel zu Euergetes endet immerhin mit einer schönen Zusammenstellung namentlich bekannter Höflinge jener Zeit. ${ }^{101}$ Der König starb wahrscheinlich 120 v. Chr., wie Roller zutreffend angibt, ohne freilich auf die bestehenden Datierungsprobleme hinzuweisen. ${ }^{102}$

Im Ganzen zutreffend ist aber sicher Rollers Ansicht, dass Pontos unter Mithradates V. zur größten Territorialmacht in Kleinasien avancierte (89). Doch warum dieser Herrscher weniger „expansionist“ als sein Vater Pharnakes gewesen sei, bleibt unklar. Mir jedenfalls erscheint die intensive Nutzung bald militärischer, bald diplomatischer Mittel, zu denen vor allem auch dynastische Ehen gehörten, als konsequente Fortsetzung der Politik all seiner Vorgänger seit Mithradates I. Ktistes. Und bereits unter Pharnakes I. wurde der pontische Hegemonialanspruch mit einer Freundschaft zu Rom spannungs-, aber erfolgreich verbunden. Daran hielten alle seine Nachfolger bis in die frühen Herrschaftsjahre Eupators fest. ${ }^{103}$

\footnotetext{
${ }^{100}$ ROLLER 2020, 86-90. Phrygien wird grob als eine Einheit betrachtet, die mal zu Pergamon gehört, mal von Pontos eingefordert wird, mal von nicht spezifizierten Galatern bewohnt wird. Auch die letztgenannten erscheinen - wiederum - als undifferenzierte Einheit, für die ein halbes Jahrhundert später pauschal von einer „strong alliance“ mit Mithradates VI. gesprochen wird (88). Fraglich ist auch die Behauptung, dass das Reich „almost to the Aegean" gereicht habe (89).

${ }^{101}$ ROLLER 2020, 90-93. Ergänze z.B. OLSHAUSEN 1974.

102 ROLler 2020, 92; vgl. 101f. Zur Diskussion siehe DE CALlATAŸ 1997, 235-242; BALlESTEROS PASTOR 2013, 82-87; abweichend datiert z.B. RYAN 2001 auf 120 v.Chr.

${ }^{103}$ ROLLER 2020, 89.
} 


\section{Bibliographie}

AlIDOUST 2020 = Alidoust, Fuad 2020: Natio molestissima. Römerzeitliche Perserbilder von Cicero bis Ammianus Marcellinus. Gutenberg.

Allen 1983 = Allen, Reginald E. 1983: The Attalid Kingdom. A Constitutional History, Oxford.

Avram 2011 = Avram, Alexandru 2011: Notes épigraphiques (I), Pontica 44, 137-140.

AVRAM 2016 = Avram, Alexandru 2016: Sur la date du traité entre Pharnace et Chersonèse Taurique, in J.Ch. Couvenhes (Hg.), La symmachia comme pratique du droit international dans le monde grec. D'Homère à l'époque hellénistique, Besançon, 213-237.

Ballesteros Pastor 2008 = Ballesteros Pastor, Luis 2008: Cappadocia and Pontus. Client Kingdoms of the Roman Republic from the Peace of Apamea to the Beginning of the Mithridatic Wars (188-89 B.C.), in Coşkun 2008, 45-63.

Ballesteros Pastor 2008/18 = Ballesteros Pastor, Luis 2008/18: Pharnakes I, King of Pontos, in Coşkun, APR s.v.

BALlesteros PASTOR 2011A = Ballesteros Pastor, Luis 2011a: Xerxes redivivus. Mitrídates, rey de Oriente frente a Grecia, in J.M. Cortés et al. (eds.), Grecia ante los Imperios. Actas de la V Reunión de Historiadores del Mundo Griego Antiguo (Carmona 2009), Sevilla, 253-262.

Ballesteros Pastor 2011B = Ballesteros Pastor, Luis 2011b: El relato sobre hipsicratea (Pomp. 32.7-8) y la imagen de mitrídates en plutarco, in J.M. Candau, F.J. González und A.L. Chávez (Hgg.), Plutarco Transmissor, Sevilla, 113-122.

BAllesteros Pastor 2012 = Ballesteros Pastor, Luis 2012: Los herederos de Artabazo. La satrapía de Dascilio en la tradición de la dinastía Mitridátida, Klio 112, 366-379.

Ballesteros Pastor 2013 = Ballesteros Pastor, Luis 2013: Pompeyo Trogo, Justino y Mitrídates. Comentario al Epítome de las Historias Filípicas (37,1,6-38,8,1), Göttingen.

Ballesteros Pastor 2015 = Ballesteros Pastor, Luis 2015: Los príncipes del Ponto. La política onomástica de Mitrídates como factor de propaganda dinástica, $R E A 117,425$ 445.

BAllesteros PASTOR 2016 = Ballesteros Pastor, Luis 2016: The Satrapy of Western Armenia in the Mithridatid Kingdom, in Victor Cojocaru und Alexander Rubel (Hgg.), Mobility in Research on the Black Sea Region, Cluj-Napoca, 273-287.

Ballesteros Pastor 2017 = Ballesteros Pastor, Luis 2017: Pharnaces II and his Title "King of Kings", AWE 16, 297-303.

Ballesteros Pastor 2018 = Ballesteros Pastor, Luis 2018: De rey del Ponto a rey de reyes. El imperio de Mitrídates Eupátor en el contexto del Oriente tardo-helenístico, in Lia R. Cresci und Francesca Gazzano (Hgg.), De Imperiis. L'idea di impero universale e la successione degli imperi nell'Antichità, Rom, 137-170. 
BAllesteros Pastor 2020A = Ballesteros Pastor, Luis 2020a: Quien da primero, da dos veces. Intrigas dinásticas en el ascenso al poder de Mitrídates Eupátor, Athenaeum $108.2,390-407$

Ballesteros Pastor 2020B = Ballesteros Pastor, Luis 2020b: The Origins of the Ariobarzanid Dynasty, Gephyra 20,61-78.

BAllesteros Pastor 2021 = Ballesteros Pastor, Luis 2021a: The Return of the King: Pharnakes II and the Achaimenid Tradition, in Coşkun 2021a, 183-198.

BALlesteros PASTOR IM ERSCHEINEN = Ballesteros Pastor, Luis im Erscheinen: The Land of the Sun and the Moon: An Interpretation of the Emblem on Royal Pontic Coins, demnächst in John Boardman, Alexandru Avram, James Hargrave und Alexandr Podossinov (Hgg.), Connecting East and West. Studies Presented to Prof. Gocha R. Tsetskhladze, Leuven 2021, Kapitel 5.

BEKKER-Nielsen 2006 = Bekker-Nielsen, Tønnes (Hg.) 2006: Rome and the Black Sea Region: Domination, Romanisation, Resistance, Aarhus.

BICKERMAN 1980 = Bickerman, Elias 1980: Chronology of the Ancient World, 2. Aufl. London.

BIFFI 2010 = Biffi, Nicola 2010: Scampoli die Mithridatika nella Geografia di Strabone, Bari.

Bosworth Und Wheatley 1998 = Bosworth, A. Brian und Wheatley, Patrick V. 1998: The Origin of the Pontic House, JHS 118, 155-164.

BRAund 2004 = Braund, David 2004: King Scribonius, in V. Chržanovskij u.a. (Hgg.), Bosporskij Fenomen (Das Bosporanische Phänomen), Teil 1, Sankt Petersburg, 81-87.

BRAUnd und Stolba 2021 = Braund, David, Peter, Ulrike und Stolba, Vladimir (Hgg.) 2021: Environment and Habitation around the Ancient Black Sea, Berlin.

BRAUND UND DAN IM ERSCHEINEN = Braund, David und Dan, Anca (Hgg.) im Erscheinen: Mithridates and the Pontic Kingdom (Collection Varia Anatolica), Paris.

BRAUND, DAN UND GEHRKE IM ERSCHEINEN = Braund, David, Dan, Anca und Gehrke, Hans-Joachim (Hgg.) im Erscheinen: The Charisma of the King: New insights into Mithridates VI Eupator's rule and legitimation of Rule (Studies in Ancient Monarchies), Stuttgart.

Burcu ErciYAs 2006 = Burcu Erciyas, Deniz 2006: Wealth, Aristocracy and Royal Propaganda under the Hellenistic Kingdom of the Mithradatids in the Central Black Sea Region of Turkey, Leiden.

BursteIn $1980=$ Burstein, Stanley M. 1980: The Aftermath of the Peace of Apamea. Rome and the Pontic War, AJAH 5, 1-12.

CANEPA 2017 = Canepa, Matthew 2017: Rival Images of Iranian Kingship and Persian Identity in Post-Achaemenid Western Asia, in Strootman und Versluys 2017, 201-222.

CANEPA 2020 = Canepa, Matthew 2020: The Iranian Expanse: Transforming Royal Identity Through Architecture, Landscape, and the Built Environment, 550 BCE - 642 CE,

http://www.fera-journal.eu 
Oakland, CA.

CARNEY 2013 = Carney, Elizabeth D. 2013: Arsinoë of Egypt and Macedon. A Royal Life, Oxford.

Chaniotis 2018 = Chaniotis, Angelos 2018: Age of Conquests. The Greek World from Alexander to Hadrian, Cambridge, MA.

ChruBASIK 2013 = Chrubasik, Boris 2013: The Attalids and the Seleukid Kings, 281-175 $\mathrm{BC}$, in Peter Thonemann (Hg.), Attalid Asia Minor. Money, International Relations, and the State, Oxford, 83-119.

CoşKun APR = Coşkun, Altay APR: Amici Populi Romani. Prosopographie der auswärtigen Freunde Roms = Prosopography of the Foreign Friends of Rome, Version 01-10, Trier 2007-2008 und Waterloo, ON 2010-2020. URL: http://www.altaycoskun.com/apr.

CoşKun 2011A = Coşkun, Altay 2011a: Galatians and Seleucids: A Century of Conflict and Cooperation, in Kyle Erickson und Gillian Ramsey (Hgg.), Seleucid Dissolution: The Sinking of the Anchor, Wiesbaden, 85-106.

CoşKun 2011B = Coşkun, Altay 2011b: Die Stratios-Mission des Jahres 167 v. Chr., ein später Einschub in den Polybios-Text $(30,2,6)$ und die dynastische Erbfolge der späteren Attaliden, Historia 60, 94-114.

CoşKun 2013 = Coşkun, Altay 2013: Von Anatolia bis Inscriptions of Ankara: Zwanzig Jahre Forschungen zum antiken Galatien (1993-2012), Anatolica 39, 69-95.

CoşKun 2014A = Coşkun, Altay 2014a: Latène-Artefakte im hellenistischen Kleinasien: ein problematisches Kriterium für die Bestimmung der ethnischen Identität(en) der Galater, IstMitt 64, 129-162.

CoşKun 2014B = Coşkun, Altay 2014b: Kastor von Phanagoreia, Präfekt des Mithradates und Freund der Römer, in Nikolai Povalahev (Hg.), Phanagoreia und darüber hinaus ... - Festschrift für Vladimir Kuznetsov, Göttingen, 131-138.

CoșKun 2016A = Coşkun, Altay 2016a: Heinz Heinen und die Bosporanischen Könige Eine Projektbeschreibung, in Victor Cojocaru und Alexander Rubel (Hgg.), Mobility in Research on the Black Sea. (Iaşi, July 5-10, 2015), Cluj-Napoca, 51-71.

CoșKun 2016B = Coşkun, Altay 2016b: Philologische, genealogische und politische Überlegungen zu Ardys und Mithradates, zwei Söhnen des Antiochos Megas (Liv. 33,19,9), Latomus 75, 849-861.

CoşKun 2018A = Coşkun, Altay 2018a: Brogitaros and the Pessinus-Affair. Some Considerations on the Galatian Background of Cicero's Lampoon against Clodius in 56 BC (Harusp. Resp. 27-29), Gephyra 15, 117-131. URL: https://dergipark.org.tr/en/pub/gephyra/issue/31130.

CoşKun 2018B = Coşkun, Altay 2018b: The War of Brothers, the Third Syrian War, and the Battle of Ankyra (246-241 BC): a Re-Appraisal, in Kyle Erickson (Hg.), The Seleukid Empire, 281-222 BC. War within the Family, Swansea, 197-252.

CoşKun 2019A = Coşkun, Altay 2019a: Epilogue, in Coşkun und Engels 2019, 457-479.

http://www.fera-journal.eu 
CoşKun 2019B = Coşkun, Altay 2019b: The Date of the Revolt of Asandros and the Relations between the Bosporan Kingdom and Rome under Caesar, in Margret Nollé, Peter M. Rothenhöfer, Gisela Schmied-Kowarzik, Hertha Schwarz und Hans Christoph von Mosch (Hgg.), Panegyrikoi Logoi. Festschrift für Johannes Nollé zum 65. Geburtstag, Bonn, 125-146.

CoşKun 2019C = Coşkun, Altay 2019c: Chersonesos Taurike, Asandros and Rome - A New Interpretation of the Embassy of C. Julius Satyrus to Rome, 46 BC (IOSPE I² 691), in Alice Bencivenni, Alessandro Cristofori, Federicomaria Muccioli und Carla Salvaterra (Hgg.), PHILOBIBLOS - Scritti in onore di Giovanni Geraci, Mailand, 281-306.

CoşKun 2019/20 = Coşkun, Altay 2019/20: The Course of Pharnakes II's Pontic and Bosporan Campaigns in 48/47 BC, Phoenix 73.1-2, 2019 (2020), 86-113.

CoşKun 2021A = Coşkun, Altay 2021a: Ethnic Constructs, Royal Dynasties and Historical Geography around the Black Sea Littoral. With the assistance of Joanna Porucznik and Germain Payen, Geographica Historica, Stuttgart 2021.

CoşKun 2021B = Coşkun, Altay 2021b: Deiotaros Philorhomaios, Pontos und Kolchis, Coşkun 2021a, 233-263.

CoşKun 2021C = Coşkun, Altay 2021c: Pompeius und die ,elf Städte' der Provinz Pontus, Coşkun 2021a, 265-285.

CoşKun 2021D = Coşkun, Altay 2021d: Seleucid Throne Wars. Resilience and Disintegration of the Greatest Successor Kingdom from Demetrius I to Antiochus VII, in Andrea Berlin und Paul Kosmin (Hgg.), The Middle Maccabees from the Death of Judas through the Reign of John Hyrcanus (161-104 BC). New Archaeological and Historical Perspectives, Atlanta, GA 2021, 269-291.

CoşKun 2021E = Coşkun, Altay 2021e: I Galati in Asia Minore nel III secolo a.C., in Cinzia Bearzot, Franca Landucci Gattinoni und Giuseppe Zecchini (eds.), I Celti e il Mediterraneo, Mailand 2021, 136-164.

COŞKUN IM ERSCHEINEN A = Coşkun, Altay im Erscheinen a: Die Teilung ,Armeniens“ durch Caesar und die Entstehung ,Kleinarmeniens', demnächst in Orbis Terrarum 19, 2021.

COŞKUN IM ERSCHEINEN B = Coşkun, Altay im Erscheinen b: Von Amaseia nach Sinope. Die Expansion des Pontischen Reiches unter Pharnakes I., demnächst in Ricerche Ellenistiche 2.

COŞKUN IM ERSCHEINEN C = Coşkun, Altay (Hg.) im Erscheinen c: Galatian Victories and Other Studies into the Agency and Identity of the Galatians in the Hellenistic and EarlyRoman Periods (Colloquia Antiqua 33), Leuven.

COŞKUN IM ERSCHEINEN D = Coşkun, Altay im Erscheinen d: Die geopolitische Bedeutung der Flüsse Tanais und Kalykadnos in den Territorialklauseln des Friedensvertrags von Apameia (Liv. 38,38,4.9), demnächst in Orbis Terrarum 20, 2022.

COŞKUN IM ERSCHEINEN E = Coşkun, Altay im Erscheinen e: The Bosporan Kings inbetween the Mithridatic Tradition and Friendship with Rome: the Usurpation of 
Asandros Revisited, demnächst in David Braund, Angelos Chaniotis und Elias Petropoulos (Hgg.), Roman Pontos, Athen.

COŞKUN IM ERSCHEINEN F = Coşkun, Altay im Erscheinen f. Expansion und Dynastische Politik in Pontos: Zwei neue Ären unter Pharnakes I., demnächst in Historia.

CoŞKUn In Begutachtung A = Coşkun, Altay in Begutachtung a: Von Kios nach Kimiata. Überlegungen zur Gründung des Mithradatidenreiches.

CoşKun In Begutachtung B = Cosskun, Altay in Begutachtung b: The Chronology of the Asylia Dossier from Kos Revisited in Light of Some Recent Epigraphic Discoveries.

COŞKUN IN VORBEREITUNG = Coşkun, Altay in Vorbereitung: Polygamy and Queenship under Antiochos II - Implications of the Basilissa Title (or the Lack thereof) at the Early Seleukid Court.

CoşKun und Engels 2019 = Coşkun, Altay und Engels, David (Hgg.) 2019: Rome and the Seleukid East. Selected Papers from Seleukid Study Day V, Brussels, 21-23 Aug. 2015, Brüssel.

CoşKun Und HeInen 2004 = Coşkun, Altay und Heinen, Heinz 2004: Amici populi Romani. Das Trierer Projekt 'Roms auswärtige Freunde' stellt sich vor, AncSoc 34, 45-75.

CoşKun Und STERn 2021 = Coşkun, Altay und Stern, Gaius 2021: Dynamis in Rome? Revisiting the South Frieze of the Ara Pacis Augustae, in Coşkun 2021a, 199-230.

D'Agostini 2016 = D'Agostini, Monica 2016: The Multicultural Ties of the Mithridatids: Sources, Tradition and Promotional Image of the Dynasty of Pontus in $4^{\text {th }}-3^{\text {rd }}$ Centuries B.C. With an Appendix on the Earliest Issues of Pontic Coins and Laodice III's Dowry, Aevum 90.1, 83-96.

DE CALLATAŸ 1997 = de Callataÿ, François 1997: L'histoire des guerres mithridatiques vue par les monnaies, Louvain-la-Neuve.

DE CAllatä̈ 2009 = de Callataÿ, François 2009: The First Royal Coinages of Pontos (from Mithridates III to Mithridates V), in Jakob M. Højte (ed.), Mithridates VI and the Pontic Kingdom, Aarhus, 63-94.

DREYER 2005 = Dreyer, Boris 2005: Rom und die griechischen Polisstaaten an der westkleinasiatischen Küste in der zweiten Hälfte des zweiten Jahrhunderts v. Chr. Hegemoniale Herrschaft und lokale Eliten im Zeitalter der Gracchen, in Altay Coşkun (Hg.), Roms auswärtige Freunde in der späten Republik und im frühen Prinzipat, Göttingen, 55-74.

DREYER 2007 = Dreyer, Boris 2007: Die römische Nobilitätsherrschaft und Antiochos III. (205 bis 188 v. Chr.), Hennef.

ECKSTEIN 2008 = Eckstein, Arthur M. 2008: Rome Enters the Greek East. From Anarchy to Hierarchy in the Hellenistic Mediterranean, 230-170 B.C., Malden, MA.

EHLING 2008 = Ehling, Kai 2008: Untersuchungen zur Geschichte der späten Seleukiden (16463 v. Chr.). Vom Tode des Antiochos IV. bis zur Einrichtung der Provinz Syria unter Pompeius, Stuttgart. 
ENGELS 2014 = Engels, David 2014: „Je veux être calife à la place du calife“? Überlegungen zur Funktion der Titel „Großkönig“" und „König der Könige“ vom 3. zum 1. Jh. v. Chr., in Victor Cojocaru, Altay Coskun und Madalina Dana (Hgg.), Interconnectivity in the Mediterranean and Pontic World during the Hellenistic and the Roman Periods, Cluj-Napoca, 333-362.

ENGELS 2017 = Engels, David 2017: Studies on the Seleukid Empire. Between East and West, Leuven.

ENGELS 1999 = Engels, Johannes 1999: Augusteische Oikumenegeographie und Universalhistorie im Werk Strabons von Amaseia, Stuttgart.

ERRINGTON 2014 = Errington, R. Malcom 2014: Rom und das Schwarze Meer im 2. Jh. v. Chr., in Victor Cojocaru und Christof Schuler (Hgg.), Die Außenbeziehungen pontischer und kleinasiatischer Städte in hellenistischer und römischer Zeit. Akten einer deutschrumänischen Tagung in Constanta, 20.-24. September 2010, Stuttgart 2014, 37-44.

ERRINGTON 2020 = Errington, R. Malcom 2020: Die Staatsverträge des Altertums. Bd. 4: Die Verträge der griechisch-römischen Welt von ca. 200 v. Chr. bis zum Beginn der Kaiserzeit. Unter Mitarbeit von Isabelle Mossong, München.

FACELLA 2006 = Facella, Margherita 2006: La dinastia degli Orontidi nella Commagene ellenistico-romana, Pisa.

FLEISCHER 2009 = Fleischer, Robert 2009: The Rock-Tombs of the Pontic Kings in Amaseia, in Højte 2009a, 109-119.

FLEISCHER 2017 = Fleischer, Robert 2017: Die Felsgräber der Könige von Pontos in Amasya, Tübingen.

GABELKo 2005 = Gabelko, Oleg L. 2005: Critical Notes on the Chronology and Dynastic History of the Kingdom of Pontus, VDI 254.4, 128-157 (Russian, with English abstract on p. 157).

GABELKo 2009 = Gabelko, Oleg L. 2009: The Dynastic History of the Hellenistic Monarchies in Asia Minor According to the Chronography of George Synkellos, in Højte 2009a, 47-61.

GABELKO 2013 = Gabelko, Oleg L. 2013: A Historical and Epigraphic Commentary on Hypsicrateia's Epitaph, in A. Mehl, A.V. Makhlayuk und Oleg L. Gabelko (Hgg.), Ruthenia Classica Aetatis Novae. A Collection of Works by Russian Scholars in Ancient Greek and Roman History, Stuttgart, 173-184.

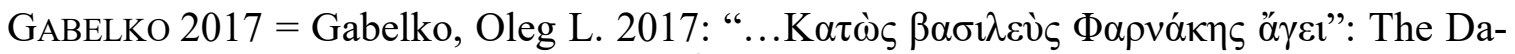
ting and Interpretation of the IOSPE I ${ }^{2} 402$ Reconsidered (in Russian), in ARCHON Ancient Relics of Chersonese: Openings to Notions. Proceedings of the International Academic Conference, Sevastopol, 10-12 October 2017, Sevastopol, 47-52.

GABELKo und ZavojKin 2004 = Gabelko, Oleg L. und Zavojkin, Alexey A. 2004: Once again about the Bithynian-Pontic-Bosporan Era (in Russian), in Bosporski fenomen I, Sankt Petersburg, 74-81. 
GAJDUKEVIČ 1971 = Gajdukevič, Viktor F. 1971: Das Bosporanische Reich, 2. Aufl. Berlin.

GALlOTTA $2010=$ Gallotta, Stefania 2010: Appunti per una storia della lega del Nord, in F. Gazza und L. Santi Amantini (Hgg.), Incontri e conflitti. Ripensando la colonizzazione greca, Rom, 93-100.

GHIŢA 2011 = Ghiţa, Christian E. 2011: Nysa - A Seleucid Princess in an Anatolian Context, in Kyle Erickson und Gillian Ramsey (Hgg.), Seleucid Dissolution: The Sinking of the Anchor, Wiesbaden, 107-116.

GOUKOWSKY 2003 = Goukowsky, Paul 2003: Appien, Histoire Romaine. Bd. 7: Livre XII: La Guerre de Mithridate, 2. Aufl. Paris.

GRAINGER 1995 = Grainger, John D. 1995: The Campaign of Cn. Manlius Vulso in Asia Minor, $A S$ 45, 23-42.

GRAINGER 1997 = Grainger, John D. 1997: A Seleukid Prosopography and Gazetteer, Leiden.

GRAINGER 2014 = Grainger, John D. 2014: The Rise of the Seleukid Empire (323-223 BC), Barnsley.

GRAINGER 2015A = Grainger, John D. 2015a: The Seleukid Empire of Antiochus III (223187 BC), Barnsley.

GRAINGER 2015B = Grainger, John D. 2015b: The Fall of the Seleukid Empire (187-75 BC), Barnsley.

HABICHT 1997 = Habicht, Christian 1997: Athens from Alexander to Antony, transl. Deborah Lucas Schneider, Cambridge, MA 1997.

HÄMMERLING 2019 = Hämmerling, Robin 2019: Zwischen dynastischem Selbstbild und literarischem Stereotyp. Königinnen der Seleukiden und der Mittelmächte Kleinasiens, Rahden in Westfalen.

HANNESTAD 2020 = Hannestad, Lise 2020: Nicator. Seleucus I and His Empire, Aarhus.

HANSEN 1971 = Hansen, Esther V. 1971: The Attalids of Pergamon, 2. Aufl. Ithaca.

HARRISON 1982 = Harrison, Cynthia M. 1982: Persian Names on Coins of Northern Anatolia, Journal of Near Eastern Studies 41, 181-194.

HEAD 1911 = Head, Barclay V. 1911: Historia Numorum. A Manual of Greek Numismatics, 2. Aufl. Oxford.

HECKEL 2006 = Heckel, Waldemar 2006: Who's Who in the Age of Alexander the Great, Malden, MA.

HECKEL 2020 = Heckel, Waldemar 2020: In the Path of Conquest. Resistance to Alexander the Great, Oxford.

HeINEMANN 2010 = Heinemann, Uwe 2010: Stadtgeschichte im Hellenismus. Die lokalhistoriographischen Vorgänger und Vorlagen Memnons von Herakleia, München. 
HEINEN 2001 = Heinen, Heinz 2001: Die mithradatische Tradition der bosporanischen Könige - ein mißverstandener Befund, in Klaus Geus und Klaus Zimmermann (Hgg.), Punica - Libyca-Ptolemaica. Festschrift für Werner Huß zum 65. Geburtstag dargebracht von Schülern, Freunden und Kollegen, Leuven, 355-370 = Heinen 2006b, 343358.

HEINEN 2005 = Heinen, Heinz 2005: Die Anfänge der Beziehungen Roms zum nördlichen Schwarzmeerraum. Die Romfreundschaft der Chersonesiten (IOSPE I $\left.{ }^{2}, 402\right)$, in Altay Coşkun (Hg.), Roms auswärtige Freunde in der späten Republik und im frühen Prinzipat, Göttingen, 31-54.

HEINEN 2006A = Heinen, Heinz 2006a: Antike am Rande der Steppe. Der nördliche Schwarzmeerraum als Forschungsaufgabe, Stuttgart.

HEINEN 2006B = Heinen, Heinz 2006b: Vom hellenistischen Osten zum römischen Westen. Ausgewählte Schriften zur Alten Geschichte, hg. von Andrea Binsfeld und Stefan Pfeiffer, Stuttgart.

HEINEN 2008A = Heinen, Heinz 2008a: Romfreunde und Kaiserpriester am Kimmerischen Bosporos. Zu neuen Inschriften aus Phanagoreia, in Altay Coşkun (Hg.), Freundschaft und Gefolgschaft in den auswärtigen Beziehungen der Römer (2. Jh. v. Chr. - 1. Jh. n. Chr.), Frankfurt a.M. 2008, 189-208.

HEINEN 2008B = Heinen, Heinz 2008b: La tradition mithridatique des rois du Bosphore, de Rostovtzeff à l'historiographie soviétique, in Jean Andreau und Wladimir Berelowitch (Hgg.), Michel Ivanovitch Rostovtzeff, Bari, 137-152.

HEINEN 2012 = Heinen, Heinz 2012: Hypsikrateia/Hypsikrates: Travestie aus Liebe. König Mithradates Eupators Page und eine neue griechische Inschrift aus Phanagoreia/ Rußland, JAWG 2012 (2014), Berlin, 215-238.

HøJTE 2005 = Højte, Jakob M. 2005: The Date of the Alliance between Chersonesos and Pharnakes (IOSPE $\left.\mathrm{I}^{2}, 402\right)$ and Its Implications, in Vladimir F. Stolba und Lise Hannestad (Hgg.), Chronologies of the Black Sea Area in the Period c. 400-100 BC, Aarhus, 137-152.

HøJTE 2009A = Højte, Jakob M. (ed.) 2009a: Mithridates VI and the Pontic Kingdom, Aarhus.

HøJTE 2009B = Højte, Jakob M. 2009b: The Death and Burial of Mithridates VI, in Højte 2009a, 121-130.

IVANTCHIK UND TOKHTAS'Ev 2011 = Ivantchik, Askold I. und Tokhtas'ev, Sergey R. 2011: Queen Dynamis and Tanais, in Ewdoksia Papuci-Władyka et al. (Hgg.), Recent Research on the Northern and Eastern Black Sea in Ancient Times. Proceedings of the International Conference, $21^{\text {st }}-26^{\text {th }}$ April 2008, Krakau, 163-173.

JACOBS 2002 = Jacobs, Bruno 2002: Die Galerien der Ahnen des Königs Antiochos I. von Kommagene auf dem Nemrud Dağ 1 , in Jakob M. Højte (ed.), Images of Ancestors, Aarhus, 75-88. 
JAJLENKO 2010 = Jajlenko, Valerij P. 2010: Tysjačeletnij Bosporskij Reich. Istorija i èpigrafika Bospora VI v. do n.e. - V v.n.e. (Das Tausendjährige Bosporanische Reich. Geschichte und Inschriften des Bosporos vom 6. Jh. v. Chr. bis zum 5. Jh. n. Chr.), Moskau.

JONES 1993 = Jones, Chistopher P. 1993: The Decree of Illion in Honor of a King Antiochus, GRBS 34, 73-92.

JUNG-KAISER 2016 = Jung-Kaiser, Ute 2016: Mithridates, in Peter von Möllendorff, Annette Simonis and Linda Simonis (Hgg.), Figures of Antiquity and Their Reception in Art, Literature and Music (BNP Suppl. 7), Leiden, 433-437.

KISTLER 2009 = Kistler, Erich 2009: Funktionalisierte Keltenbilder. Die Indienstnahme der Kelten zur Vermittlung von Normen und Werten in der hellenistischen Welt, Frankfurt.

KozlovsKaya 2017 = Kozlovskaya, Valeriya (Hg.) 2017: Northern Black Sea in Antiquity: Networks, Connectivity, and Cultural Interactions, Cambridge.

Kosmin 2018 = Kosmin, Paul J. 2018: Time and Its Adversaries in the Seleucid Empire, Cambridge, MA.

KuZNETSOv 2017 = Kuznetsov, Vladimir (Hg.) 2017: Phanagoreia, Moskau.

LAFLI UND CHRISTOF 2011 = Lafl, Ergün und Christof, Eva 2011: Der kaiserzeitliche Tempel von Asartepe / Kimistene in der Chora des paphlagonischen Hadrianopolis - Ergebnisse der Prospektion von 2005, IstMitt 61, 233-286.

Lerouge-Cohen 2017 = Lerouge-Cohen, Charlotte 2017: Persianism in the Kingdom of Pontic Kappadokia. The Genealogical Claims of the Mithridatids, in Strootman und Versluys 2017, 223-234.

LESCHHORN 1993 = Leschhorn, Wolfgang 1993: Antike Ären. Zeitrechnung, Politik und Geschichte im Schwarzmeerraum und in Kleinasien nördlich des Tauros, Stuttgart.

MACDONALD 2005 = MacDonald, David 2005: An Introduction to the History and Coinage of the Kingdom of the Bosporus. Including the Coinage of Panticapaeum (with "Apollonia" and "Myrmecium"), Phanagoria, Gorgippia, Sindicus Limen or the Sindoi, Nymphaeum, Theodosia, and the Kings of the Cimmerian Bosporus, Lancaster.

MadSEn 2020 = Madsen, Jesper M. 2020: From Trophy Towns to City-States. Urban Civilization and Cultural Identities in Roman Pontus, Philadelphia.

MAKAROV 2017 = Makarov, Igor 2017: III 103. Chersonesos. Treaty between Chersonesos and Pharnakes I of Pontus, 179 B.C.E. (?), IOSPE. Ancient Inscriptions of the Northern Black Sea. URL: https://iospe.kcl.ac.uk/3.103.html (23. Januar 2021).

MAREK 1993 = Marek, Christian 1993: Stadt, Ära und Territorium in Pontus-Bithynia und Nord-Galatia, Tübingen.

MATYSZAK 2008/15 = Matyszak, Philip 2008/15. Mithridates the Great. Rome's Indomitable Enemy, Barnsley 2008, repr. 2015.

MATYSZAK 2020 = Matyszak, Philip 2020. Greece against Rome. The Fall of the Hellenistic Kingdoms 250-31 BC, Barnsley. 
MAYOR 2010 = Mayor, Adrienne 2010: The Poison King: the Life and Legend of Mithradates, Rome's Deadliest Enemy, Princeton.

MCAuley 2019 = McAuley, Alex 2019: L'ombre lointaine de Rome: La Cappadoce à la suite de la paix d'Apamée, in Coşkun und Engels 2019, 309-332.

MCDonAld 1967 = McDonald, Alexander H. 1967: The Treaty of Apamea (188 B.C.), JRS 57, 1-8.

MCGing 1986A = McGing, Brian C. 1986a: The Foreign Policy of Mithridates Eupator, King of Pontus, Leiden.

MCGING 1986B = McGing, Brian C. 1986b: The Kings of Pontus: Some Problems of Identity and Date, $R h M 129,248-259$.

MCGING 2014 = McGing, Brian C. 2014: Iranian Kings in Greek Dress? Cultural Identity in the Mithradatid Kingdom of Pontos, in Tønnes Bekker-Nielsen (Hg.), Space, Place and Identity in Northern Anatolia, Göttingen, 21-37.

MiCHELS 2009 = Michels, Christoph 2009: Kulturtransfer und monarchischer 'Philhellenismus'. Bithynien, Pontos und Kappadokien in hellenistischer Zeit, Göttingen.

MielCZAREK 1999 = Mielczarek, Mariusz 1999: The Army of the Bosporan Kingdom, übersetzt von Nicholas Sekunda, Łódź.

MiLETA 2014 = Mileta, Christian 2014: Seleukos IV. Eupator - ein zu normaler Herrscher?, in Christophe Feyel und Laetitia Graslin-Thomé (Hgg.), Le projet politique d'Antiochos $I V$, Nancy, 165-180.

Mitchell 1993 = Mitchell, Stephen 1993: Anatolia: Land, Men, and Gods in Asia Minor, Bd. 1: The Celts in Anatolia and the Impact of Roman Rule, Oxford.

MitCHELl 2003 = Mitchell, Stephen 2003: The Galatians: Representation and Reality, in Andrew Erskine (Hg.), A Companion to the Hellenistic World, Oxford, 280-292.

MordVINTSEVA 2021 = Mordvintseva, Valentina 2021: ,Iraner" und ,Sarmaten“ in der Weltsicht Michael Rostovtzeffs, in Coşkun 2021a, 25-43.

MuCCIOLI 2013 = Muccioli, Federicomaria 2013: Gli epiteti ufficiali dei re ellenistici, Stuttgart.

MüLLER 2010 = Müller, Christel 2010: D'Olbia à Tanaïs. Territoires et réseaux d'échanges dans la mer Noire septentrionale aux époques classiques et hellénistiques, Bordeaux.

MüLlER 2012 = Müller, Christel 2012: Panticapée, polis et capitale: la place des cités dans le royaume du Bosphore de Spartokos I à Mithridate VI, in Christophe Feyel u.a. (Hgg.): Communautés locales et pouvoir central dans l'Orient hellénistique et romain, Nancy, 139-159.

MÜLLER UND MALKIN 2012 = Müller, Christel und Malkin, Irad 2012: Vingt ans d'ethnicité: bilan historiographique et application du concept aux études anciennes, in Laurent Capdetrey und Julien Zurbach (Hgg.): Mobilités grecques. Mouvements, réseaux, contacts en Méditerranée, de l'époque archaïque à l'époque hellénistique, Bordeaux, 25-37. 
OGDEN 2017 = Ogden, Daniel 2017: The Legend of Seleucus. Kingship, Narrative and Mythmaking in the Ancient World, Cambridge.

OLBRYCHT 2009 = Olbrycht, Marek J. 2009: Mithridates Eupator and Iran, in Højte 2009a, 163-190.

OLBRYCHT 2019 = Olbrycht, Marek J. 2019: The Memory of the Past: the Achaemenid Legacy in the Arsakid Period, Studia Litteraria Universitatis Iagellonicae Cracoviensis 14 (Special Issue), 175-186.

OLSHAUSEN 1974 = Olshausen, Eckart 1974: Zum Hellenisierungsprozess am pontischen Königshof, AncSoc 5, 153-170.

OLSHAUSEN UND BILLER 1984 = Olshausen, Eckart und Biller, J. 1984: Historisch-geographische Aspekte der Geschichte des Pontischen und Armenischen Reiches. Teil I: TAVO $B$ 29.1, Wiesbaden.

PARFENOV 1996 = Parfenov, Vladimir N. 1996: Dynamis, Agrippa und der Friedensaltar. Zur militärischen und politischen Geschichte des Bosporanischen Reiches nach Asandros, Historia 45, 95-103.

PATON UND HABICHT 2012 = Paton, W.R., Walbank, Frank und Habicht, Christian 2012: Polybius, The Histories, Books 16-27. Translated by Paton, revised by Walbank and Habicht, Cambridge, MA.

PATTERSON 2001 = Patterson, Lee 2001: Rome's Relationship with Artaxias I of Armenia, $A H B$ 15, 2001, 154-162.

PAYEN 2016 = Payen, Germain 2016: Le traité d'Apamée et ses suites: redéfinition des frontières royales hellénistiques en Anatolie, in Hugues Berthelot u.a. (Hgg.), Vivre et penser les frontières dans le monde méditerranéen antique, Bordeaux, 107-115.

PAYEN 2019 = Payen, Germain 2019: L'influence séleucide sur les dynasties anatoliennes après le traité d'Apamée, in Coşkun und Engels 2019, 279-307.

PAYEN 2020 = Payen, Germain 2020: Dans l'ombre des empires. Les suites géopolitiques du traité d'Apamée en Anatolie, Québec.

PAYEN 2021A = Payen, Germain 2021a: Les suites de la paix d'Apamée en mer Noire, in Coşkun 2021a, 161-182.

PAYEN 2021 B = Payen, Germain 2021b: La guerre d'Eumène II et ses alliés contre Pharnace (182-179 a.C.). Problèmes et lectures géopolitiques des suites du traité d'Apamée, in Anaïs Lamesa und Giusto Traina (Hgg.), L'Anatolie, de l'époque archä̈que à Byzance (Supplément des Dialogues d'histoire ancienne 22), 157-181.

PAYEN IM ERSCHEINEN A = Payen, Germain im Erscheinen a: When Galatians Unite? A Geopolitical Evaluation of the Impact of the Alleged Galatian Unity in the Second Century BC, demnächst in CoşKUN IM ERSCHEINEN C, 193-212.

PAYEN IM ERSCHEINEN B = Payen, Germain im Erscheinen b: Le royaume artaxiade dans l'Empire séleucide: de dominé à dominant, demnächst in Altay Coşkun und Richard Wenghofer (Hgg.), Seleukid Ideology: Creation, Reception and Response. 
PERL 1968 = Perl, Gerhard 1968: Zur Chronologie der Königreiche Bithynia, Pontos und Bosporos, in János Harmatta (Hg.), Studien zur Geschichte und Philosophie des Altertums, Amsterdam, 299-330.

PetKoviĆ 2009 = Petković, Žarko 2009: Mithridates II and Antiochos Hierax, Klio 91.2, 378-383.

PETKOVIĆ 2012 = Petković, Žarko 2012: The Aftermath of the Apamean Settlement: Early Challenges to the new Order in Asia Minor, Klio 94.2, 357-365.

PrIMO 2006 = Primo, Andrea 2006: Mitridate III: problemi di cronologia e identità nella dinastia pontica, Studi Ellenistici 19, 307-329.

PrIMO 2008 = Primo, Andrea 2008: Seleuco e Mitridate Ktistes in un episodio del giovane Demetrio Poliorcete, in Studi Ellenistici 20, 410-425.

PrIMO 2009 = Primo, Andrea 2009. La storiografia sui Seleucidi da Megastene a Eusebio di Cesarea, Pisa.

PrIMO $2010=$ Primo, Andrea 2010: The Client Kingdom of Pontus between Mithridatism and Philoromanism, in Ted Kaizer und Margherita Facella (Hgg.), Kingdoms and Principalities in the Roman Near East, Stuttgart, 159-179.

REINACH 1888 = Reinach, Théodore 1888: Trois royaumes de l'Asie Mineure. Cappadoce - Bithynie - Pont, Paris.

RENDINE 2020 = Rendine, Simone 2020: The Cities of the Greek East after the First Mithridatic War. Aspects of Sulla's Financial Policy, Phasis 23, 73-92.

RICH 2015 = Rich, John 2015: Appian, Polybius and the Romans' War with Antiochus the Great: a Study in Appian's Sources and Methods, in Kathryn Welch (ed.), Appian's Roman History: Empire and Civil War, Swansea, 65-124.

RIGSBY 1996 = Rigsby, Kent J. 1996: Asylia. Territorial Inviolability in the Hellenistic World, Berkeley, CA.

ROBERT 1973 = Robert, Louis 1973: Monnaies et textes grecs, JSav 1973, 145-163. Vgl. Opera Minora Selecta, Bd. 7, Amsterdam 1990, 277-295.

ROLLER 1998 = Roller, Duane W. 1998: The Building Program of Herod the Great, Berkeley, CA.

ROLLER 2003 = Roller, Duane W. 2003: The World of Juba II and Kleopatra Selene. Royal Scholarship on Rome's African Frontier, London.

ROLLER 2004 = Roller, Duane W. 2004: Scholarly Kings, The Writings of Juba II of Mauretania, Archelaos of Kappadokia, Herod the Great, and the Emperor Claudius, Chicago.

ROLLER 2006 = Roller, Duane W. 2006: Through the Pillars of Herakles: Greco-Roman Exploration of the Atlantic, London.

ROLLER 2010 = Roller, Duane W. 2010: Eratosthenes' Geography. Fragments collected and translated, with commentary and additional material, Princeton. 
RoLler 2014 = Roller, Duane W. 2014: The Geography of Strabo, Cambridge.

ROLLER 2015 = Roller, Duane W. 2015: Ancient Geography. The Discovery of the World in Classical Greece and Rome, Cambridge.

Roller 2018A = Roller, Duane W. 2018a: A Historical and Topographical Guide to the Geography of Strabo, Cambridge.

RoLler 2018B = Roller, Duane W. 2018b: Cleopatra's Daughter and Other Royal Women of the Augustan Era, Oxford.

Roller 2019 = Roller, Duane W. (Hg.) 2019: New Directions in the Study of Ancient Geography, University Park, PA.

ROLLER $2020=$ Roller, Duane W. 2020: Empire of the Black Sea: the Rise and Fall of the Mithridatic World, Oxford.

RostovtzeFf 1919 = Rostovtzeff, Michael 1919: Queen Dynamis of Bosporus, JHS 39, 88-109.

RostowZEW 1931 = Rostowzew, Michael 1931: Skythien und der Bosporus. Bd. 1: Kritische Übersicht der schriftlichen und archäologischen Quellen, Berlin.

RUSSO 2015 = Russo, Federico 2015: L'odium regni a Roma tra realtà politica e finzione storiografica, Pisa.

RYAN 2001 = Ryan, Franz X. 2001: Die Zurücknahme Großphrygiens und die Unmündigkeit des Mithridates VI. Eupator, Orbis Terrarum 7, 99-107.

SAPRYKIN 1996 = Saprykin, Sergej J. 1996: Pontijskoe carstvo. Gosudarstvo grekov $i$ vararov v Pričernomor'e (Das Pontische Reich. Ein Staat von Griechen und Barbaren am Schwarzen Meer), Moskau.

SAPRYKIN 2002 = Saprykin, Sergej J. 2002: Bosporskoe carstvo na rubeže dvuch èpoch (Das Bosporanische Reich an der Wende zweier Epochen), Moskau.

SAPRYKIN 2005 = Saprykin, Sergey J. 2005: Thrace and the Bosporus under the Early Roman Emperors, in David Braund (ed.), Scythians and Greeks. Cultural Interactions in Scythia, Athens and Early Roman Empire (Sixth Century BC-First Century AD), Exeter, 167-175 und 216f. (notes).

SAPRYKIN UND FEDOSEEV 2009 = Saprykin, Sergey Y. und Fedoseev, Nikolay F. 2009: Epigraphica Pontica II: New Inscription of Pythodoris from Panticapaeum, VDI 270, 2009.3, 138-147.

SørEnSEN 2016 = Sørensen, Søren L. 2016: Between Kingdom and koinon. Neapolis/Neoklaudiopolis and the Pontic Cities, Stuttgart.

StOLBA 2005 = Stolba, Vladimir M. 2005: Hellenistic Chersonesos: Towards Establishing a Local Chronology, in Vladimir F. Stolba und Lise Hannestad (Hgg.), Chronologies of the Black Sea Area in the Period c. 400-100 BC, Aarhus, 153-177. 
STROBEL 1994 = Strobel, Karl 1994: „Keltensieg und Galatersieger“. Die Funktionalisierung eines historischen Phänomens als politischer Mythos der hellenistischen Welt, in Elmar Schwertheim (Hg.), Forschungen in Galatien, Bonn, 67-96.

Strobel Und Gerber 2000 = Strobel, Karl und Gerber, Christoph 2000: Tavium (Büyüknefes, Provinz Yozgat). Ein regionales Zentrum Anatoliens. Bericht über den Stand der Forschungen nach den ersten drei Kampagnen (1997-1999) (mit einem Beitrag von G. Erath), IstMitt 50, 215-265.

Strootman 2015 = Strootman, Rolf 2015: Seleucid Era, in Eshan Yarshater (Hg.), Encyclopaedia Iranica (Online), Winona Lake.

STROOTMAN 2016 = Strootman, Rolf 2016: "The Heroic Company of My Forebears": the Ancestor Galleries of Antiochos I of Kommagene at Nemrut Daği and the Role of Royal Women in the Transmission of Hellenistic Kingship, in Altay Coşkun und Alex McAuley (Hgg.), Seleukid Royal Women. Creation, Representation and Distortion of Hellenistic Queenship in the Seleukid Empire, Stuttgart, 209-230.

STROOTMAn 2019= Strootman, Rolf 2019: Antiochos IV and Rome: the Festival at Daphne (Syria), the Treaty of Apameia and the Revival of Seleukid Expansionism in the West, in Coşkun und Engels 2019, 173-215.

Strootman Und Versluys 2017 = Strootman, Rolf und Versluys, Miguel John (Hgg.) 2017: Persianism in Antiquity, Stuttgart.

TOMASCHITZ 2002 = Tomaschitz, Kurt 2002: Die Wanderungen der Kelten in der antiken literarischen Überlieferung, Wien.

ToumanofF 1963 = Toumanoff, Cyril 1963: Studies in Christian Caucasian History, Georgetown University Press.

TRACY 1992 = Tracy, Stephen V. 1992: Inscriptiones Deliacae: IG XI 713 and IG XI 1056, $\operatorname{MDAI}(A)$ 107, 303-314.

TraILl 1994 = Traill, John S. 1994: The Athenian Archon Pleistainos, ZPE 103, 109-114.

TRAINA 2017 = Traina, Giusto 2017: Rois ou dynastes? Les territories arméniens à l'époque d'Antiochos III, in Christophe Feyel und Laetitia Graslin-Thomé (Hgg.), Antiochos III et l'Orient. Actes de la rencontre franco-allemande tenue à Nancy du 6 au 8 juin 2016, Paris, 377-388.

Walbank 1979 = Walbank, Frank W. 1979: A Historical Commentary on Polybius, Bd. 3, Commentary on Books. XIX-XL, Oxford.

Wheatley 2019 = Wheatley, Pat 2009: The Besieger in Syria, 314-312 BC. Historiographic and Chronological Notes, in Pat Wheatley und Robert Hannah (Hgg.), Alexander and His Successors: Essays from the Antipodes, Claremont, CA, 323-333.

WheAtley 2020 = Wheatley, Pat 2020: Mithridates Ctistes and Demetrius Poliorcetes: Erastes and Eromenos?, in Monica D'Agostini, Edward M. Anson und Frances Pownall (Hgg.), Affective Relations and Personal Bonds in Hellenistic Antiquity: Studies in Honor of Elizabeth D. Carney, Oxford, 213-223. 
ZAVOIKINA 2014 = Zavoikina, Natalia 2014: Polis and Monarchy in the Bosporan Kingdom in the $1^{\text {st }}-3^{\text {rd }}$ Centuries AD, in Nikolai Povalahev (Hg.), Phanagoreia und darüber hinaus ... - Festschrift für Vladimir Kuznetsov, Göttingen, 317-344.

ZAVOYKINA, NovichikHIN UND Konstantinov 2018 = Zavoykina, Natal'ya V., Novichikhin, Andrey M. und Konstantinov, Vladimir A. 2018: Novaya posvyatitel'naya nadpis' Aspurga iz Gorgippii (New Dedicatory Inscription of King Aspurgos from Gorgippia), VDI 78, 2018.3, 680-692.

\section{Kontakt zum Autor:}

Dr. Altay Coşkun

Professor of Classical Studies

University of Waterloo

E-Mail: acoskun@uwaterloo.ca

\section{(cc) $\mathrm{Br}$}

Dieser Beitrag ist lizenziert unter einer Creative Commons Namensnennung - 4.0 International Lizenz. 